\title{
IMPACT OF SOME TREATMENTS ON GROWTH AND YIELDING OF PEA PLANTS GROWN UNDER SALINE RECLAIMED SOIL CONDITIONS \\ Rady, M. M. ${ }^{1}$ and Nevein A. El-Sawah ${ }^{2}$ \\ ${ }^{1}$ Botany Dept. Fac. Agric., Fayoum Univ., Egypt. \\ ${ }^{2}$ Horticulture Dept. Fac. Agric., Fayoum Univ., Egypt.
}

\begin{abstract}
The possibility of improving growth and yielding of pea plants grown under reclaimed soil conditions which having salinity level of about 5000 ppm was investigated in a private Farm, Fayoum, Egypt, during the two successive seasons of $2007 / 2008$ and $2008 / 2009$. For achievement this aim, the calcium paste treated-seeds of pea were sown in beds fecundated with ground sunflower heads. The producing pea plants were sprayed with ascorbic acid at the rates of $0,100,200,300$ and 400 $\mathrm{mgL}^{-1}$. Significant positive influences of calcium paste plus ground sunflower heads applied alone or in combination with all ascorbic acid treatments used under this study were observed on growth, yield of green pods and seeds as well as the tested chemical constituents. In comparison with untreated seeds sown in non-fecundated beds, the plants producing from the calcium paste treated-seeds sown in beds fecundated with ground sunflower heads represented significant increases in growth traits (i.e. plant height, No. of branches plant ${ }^{-1}$ and shoot dry weight plant ${ }^{-1}$ ) and green pods yield and its components (i.e. No. of green pods plant ${ }^{-1}$, pod weight and green pods yield feddan ${ }^{-1}$ ) as well as green seeds yield and its components (i.e. No. of green seeds pod $^{-1}, 100$-seed weight and green seeds yield feddan ${ }^{-1}$ ). In addition, the concentrations of some chemical constituents (i.e. total chlorophyll, total carotenoids, ascorbic acid, free proline, total soluble sugars, total phenols, N, P, K, Ca, Ca/ Na ratio, $\mathrm{Fe}, \mathrm{Mn}$ and $\mathrm{Zn}$ in shoots of plants besides, starch\% and protein $\%$ were increased with the shortage in total soluble sugars\% in green seeds). As for ascorbic acid foliar application, plants sprayed at all studied rates (i.e. 100, 200, 300 and 400 $\mathrm{mgL}^{-1}$ ) revealed significant increases in all investigated parameters (i.e. growth traits, green pods and seeds yields and their components as well as chemical constituents of plant shoots and green seeds) as compared with untreated plants. Ascorbic acid treatment at the rate of $300 \mathrm{mgL}^{-1}$ combined with calcium paste treated-seeds sown in beds fecundated with ground sunflower heads proved to be the best and may counteracted the inhibitory effects of salinity on pea plants.

In view of above mentioned results, it has been concluded that spraying pea plants "cv. Master-B", producing from calcium paste treated-seeds sown in beds fecundated with ground sunflower heads, with ascorbic acid at the rate of $300 \mathrm{mgL}^{-1}$ could be counteracted the adverse conditions particularly, salinity up to $5000 \mathrm{ppm}$ and consequently, economic green pods and seeds yield is obtainable.
\end{abstract}

Keywords: Pea, calcium paste, ascorbic acid, growth, yield, chemical constituents

\section{INTRODUCTION}

Pea (Pisum sativum L.) is one of the popular vegetable crops in Egypt, usually cultivated as a winter crop for consumption, processing and exportation as well. It acts as a rich source in protein, carbohydrates and other nutrients for human diet. Beside the trials for improving pea productivity in the old soils, others must be carried to improve the growth and yield of pea in the newly reclaimed soils (this kind of soil is saline). Pea is classified as a 
salt sensitive vegetable crop (Pasternak, 1987). It is nevertheless an important crop in new reclaimed areas in Egypt, where much of soils is saline. Saline conditions disrupt several physiological processes in plants leading to a general reduction in growth and yield (El-Saidi, 1997 and Greenway and Munns, 1980). The drastic influence of salinity on plant growth and metabolism was attributed, principally, to the enhanced $\mathrm{Na}^{+}$uptake which causes ion excess in plant tissues (Abbas et al., 1991). One of the primary effects of increasing salinity in the growth medium is the inhibition of $\mathrm{K}^{+}, \mathrm{Ca}^{2+}$ and $\mathrm{NO}_{3} 3$ uptake by plant roots (Maas, 1986). In addition, it is well established that salinity stress damages plant cells through production of reactive oxygen species including superoxide, hydrogen peroxide, hydroxyl anions and singlet oxygen (Scandalios, 1997). Efforts have been made to control salinity by technological means; reclamation, drainage, use of high leaching fractions and application of soil amendments (Abdel-Naby et al., 2001). On the other hand, some trials have been made to alleviate the disturbances in plant metabolism excreted by salinity stress. It has been suggested that ascorbic acid is one of natural and safety substances may help to overcome some of these inhibitory effects (Rady, 2006).

Ascorbic acid is an important antioxidant defense in plant cells (Foyer and Halliwell, 1976) to protect them by scavenging the reactive oxygen species. It also stimulates respiration activities, cell division and many enzymes activities (Rautenkranz et al., 1994). It has synergistic effects on growth, yield and its components as well as chemical composition of several crops under favourable and unfavourable environmental condition i.e. salinity (Ahmed et al., 2002 and 2003; Mostafa, 2004 and Rady, 2006). It has been also suggested that calcium is an important factor in maintenance of membrane integrity and ion-transport regulation. It is essential for $\mathrm{K}^{+} / \mathrm{Na}^{+}$ selectivity and membrane integrity (Epstein, 1961 and Hanson, 1984). Elevated $\mathrm{Ca}^{++}$concentrations in the nutrient solution mitigated the adverse effects of salinity by inhibition of $\mathrm{Na}^{+}$uptake (Greenway and Munns, 1980) and reduction in leakage of membranes (Leopold and Willing, 1984). LaHaye and Epstein (1969) clearly postulated that the $\mathrm{Ca}^{++} / \mathrm{Na}^{+}$interaction takes place at the plasmalemma. They suggested that $\mathrm{Na}^{+}$acted by displacing $\mathrm{Ca}^{++}$ from membranes, leading to increased membrane permeability and intracellular $\mathrm{Na}^{+}$concentrations.

Accordingly, this work was planned for studying the influence of foliar application with ascorbic acid, seed treatment by calcium paste and fecundating seed-beds with ground sunflower heads on growth, green yield and its components as well as some chemical constituents of pea plants grown under saline reclaimed soil conditions.

\section{MATERIALS AND METHODS}

During the two successive winter seasons of 2007/2008 and 2008/2009, a field trial was carried out at a private Farm, Fayoum, Egypt. This successive experimental attempt aimed to study the influence of ascorbic acid foliar application, seed treatment with calcium paste and fecundating seed-bed with ground sunflower heads on growth, green yield 
and some of its components as well as some chemical constituents of pea (Pisum sativum L.) plants cultivated in saline reclaimed soil (ca. $5000 \mathrm{mgL}^{-1}$ ). Before sowing, soil samples to $25 \mathrm{~cm}$ depth from the experimental site were collected and analyzed by the standard procedures of Jackson (1967). Results of the soil samples analysis are presented in Table (1).

Table (1): Physical and chemical properties of the selected site in both $2007 / 2008$ and 2008/2009 seasons.

\begin{tabular}{|c|c|c|}
\hline Property & $2007 / 2008$ & $2008 / 2009$ \\
\hline \multicolumn{3}{|l|}{ Physical: } \\
\hline Clay\% & 29.75 & 29.15 \\
\hline Silt\% & 20.05 & 20.45 \\
\hline Sand\% & 50.20 & 50.40 \\
\hline Soil texture & Loamy sand & Loamy sand \\
\hline \multicolumn{3}{|l|}{ Chemical: } \\
\hline $\mathrm{pH}(1: 2.5)$ & 7.850 & 7.690 \\
\hline $\mathrm{ECe}\left(\mathrm{dS} \mathrm{m} \mathrm{m}^{-1}\right)$ & 7.890 & 7.780 \\
\hline Organic matter\% & 1.190 & 1.230 \\
\hline $\mathrm{CaCO}_{3} \%$ & 8.140 & 8.060 \\
\hline Total N\% & 0.074 & 0.071 \\
\hline \multicolumn{3}{|c|}{ Available nutrients ( $\mathrm{mg} \mathrm{Kg}^{-1}$ soil): } \\
\hline $\mathrm{K}$ & 71.25 & 69.70 \\
\hline $\mathrm{P}$ & 18.07 & 18.65 \\
\hline $\mathrm{Fe}$ & 06.14 & 06.00 \\
\hline $\mathrm{Mn}$ & 04.69 & 05.04 \\
\hline$Z n$ & 01.05 & 00.96 \\
\hline $\mathrm{Cu}$ & 01.63 & 01.70 \\
\hline
\end{tabular}

Seeds of pea cv. "Master-B" obtained from agricultural research center, Giza, Egypt were sown on November 15, 2007 and 2008. A seasonal total of 200,200 and $100 \mathrm{~kg}$ feddan ${ }^{-1}$ calcium superphosphate $\left(15.5 \% \mathrm{P}_{2} \mathrm{O}_{5}\right)$, ammonium sulphate $(20.5 \% \quad \mathrm{~N})$ and potassium sulphate $\left(48 \% \quad \mathrm{~K}_{2} \mathrm{O}\right)$, respectively were applied. $\mathrm{N}$ and $\mathrm{K}_{2} \mathrm{O}$ amounts were split into two halves. One half is applied in one month after sowing and the other half applied after 15 days later while, $P$ amount applied on the surface soil at the beginning of the experiment. Recommended cultural practices for growing pea plants were followed. Treatments comprised 5 ascorbic acid rates; $0,100,200,300$ and $400 \mathrm{mgL}^{-1}$. These treatments were applied alone or in combination with seed treatment with calcium paste sown in beds fecundated with ground sunflower heads.

\section{Method of ascorbic acid application:}

Ascorbic acid at mentioned concentrations was sprayed on shoots of plants to run off, two times; 25 and 40 days after sowing. Few drops of tween20 were added to the spraying solution as a wetting agent.

\section{Method of seed treatment with calcium paste:}

\section{a. Preparation of calcium paste:}

Calcium nitrate was mixed with wheat bran (a by-product of wheat) $(1: 2 \mathrm{~W} / \mathrm{W})$ and kneaded together by using Arabic Gum solution ( $8 \%$ ) as a sticking agent to obtain paste of calcium able to remaining around seeds to longer time. 


\section{b. Treatment of seeds with calcium paste:}

Before sowing, pea seeds were covered with calcium paste through the better mixing between them. Treated seeds were allowed to dry.

Fecundating seed-bed with ground sunflower heads:

Air-dried sunflower heads (a by-product of sunflower crop) were well cleaned by washing in tap water and dried in forced-air oven at $70^{\circ} \mathrm{C}$ for $48 \mathrm{~h}$. Then, grinded in high-speed laboratory grinding mill (The Straub Company Model 4E Grinding Mill) to pass through $2.5 \mathrm{~mm}$ sieve. The ground sunflower heads were added at fair amounts in seed-beds before sowing directly as a water retainment agent in the rizhosphere.

Chemical analysis of wheat bran and ground sunflower heads were determined (Table, 2) as outlined by A.O.A.C. (1995). Moisture content was estimated at $105^{\circ} \mathrm{C} / 3 \mathrm{~h}$. Ash was estimated by aching in an electric muffle at $550^{\circ} \mathrm{C}$ for 6 hours. Total fibers (\%) were determined by an enzymatic gravimetric method as described by Prosky et al. (1988) as well as water holding capacity was measured as outlined by Eastwood (1973).

Total (2): Some constituents of wheat bran and ground sunflower heads used in the experiment (mean of the two seasons).

\begin{tabular}{|l|c|c|}
\hline Property & Wheat bran & $\begin{array}{c}\text { Ground sunflower } \\
\text { heads }\end{array}$ \\
\hline Moisture\% & 4.50 & 10.80 \\
\hline Ash\% & 5.20 & 15.36 \\
\hline Total fibers\% & 35.30 & 39.10 \\
\hline Water holding capacity (g/g) & 3.30 & 7.70 \\
\hline
\end{tabular}

The experimental design used was a split-plot design with four replicates. The main plots were calcium paste treated-seeds sown in beds fecundated with ground sunflower heads or untreated seeds sown in nonfecundating beds, and the submain plots included ascorbic acid treatments. Each experimental unit consisted of 5 rows $3 \mathrm{~m}$ long and $70 \mathrm{~cm}$ width, within row spacing $20 \mathrm{~cm}$ apart.

\section{Recorded data:}

\section{Vegetative growth traits:}

Sixty days after sowing, four plants were randomly chosen from each experimental unit and cut off at ground level and submitted to the following determinations: plant height $(\mathrm{cm})$, No. of branches plant ${ }^{-1}$ and shoot dry weight plant ${ }^{-1}(\mathrm{~g})$.

\section{Green yield and its components:}

At the proper stage of marketable green pods, harvesting was done and samples of 20 pods were randomly taken from each experimental unit and the following data were recorded: No. of green pods plant ${ }^{-1}$, green pod weight (g), No. of seeds pod-1 $^{-1}, 100$-seed weight (g), green pods yield feddan ${ }^{-1}$ (ton) and green seeds yield feddan ${ }^{-1}(\mathrm{Kg})$.

3. Chemical constituents:

Shoots of 6 randomly selected plants were collected on January 15, in both seasons (fresh seeds at the marketable stage) from each experimental unit for chemical determinations. Total chlorophyll and total carotenoids were 
extracted from fresh leaves by acetone $(80 \%)$ then; their concentrations were determined $\left(\mathrm{mgg}^{-1}\right)$ using colorimetric method as described by Arnon (1949). Total phenols were extracted from fresh shoots using $80 \%$ ethanol then, colorimetrically determined $\left(\mathrm{mgg}^{-1}\right)$ using Folin-Denis reagent by the method of Snell and Snell (1953). Ascorbic acid was determined using the dye 2,6dichlorophenol indophenol method as outlined by A.O.A.C. (1995) in fresh shoots. Starch (\%) was extracted from fresh seeds using $80 \%$ ethanol then, hydrolyzed by concentrated $\mathrm{HCl}$ then, determined by using Fehling reagent $(A+B)$ and methylene blue as an indicator (A.O.A.C., 1995). The following parameters were determined using dry matter of shoots and harvested fresh seeds. Total soluble sugars \% (in shoots and seeds) were colorimetrically determined using phosphomolybdic acid reagent according to Dubois et al. (1956). Free proline was extracted from dry matter of shoots by 5sulphosalicylic acid (3\%) then, determined colorimetrically $\left(\mathrm{mgg}^{-1}\right)$ using acid ninhydrin reagent as outlined by Bates et al. (1973). Nitrogen $\left(\mathrm{mgg}^{-1} \mathrm{dry}\right.$ matter of shoots and seeds) was colorimetrically determined by using the Orange $G$ dye according to the method of Hafez and Mikkelsen (1981) then, protein $\%$ in seeds was calculated by multiplying $\mathrm{N} \% \times 6.25$. For $\mathrm{P}, \mathrm{K}, \mathrm{Ca}$, $\mathrm{Na}, \mathrm{Fe}, \mathrm{Mn}$ and $\mathrm{Zn}$ determinations; the wet digestion of $0.1 \mathrm{~g}$ of fine dry material of shoots of each treatment was done with sulphuric and perchloric acids mixture as mentioned by Piper (1947). Phosphorus $\left(\mathrm{mgg}^{-1}\right)$ was colorimetrically estimated using chlorostannus molybdo-phosphoric blue color method in sulphuric acid system as described by Jackson (1967). Potassium and sodium $\left(\mathrm{mg} \mathrm{g}^{-1}\right)$ was determined using a Perkin-Elmer, Flame Photometer (Page et al., 1982). Calcium $\left(\mathrm{mgg}^{-1}\right)$, iron (ppm), manganese (ppm) and zinc (ppm) were determined using a Perkin-Elmer, Model 3300, Atomic absorption Spectrophotometer (Chapman and Pratt, 1961).

\section{Statistical analysis.}

All data obtained in both seasons of this study were subjected to the analysis of variance. LSD at 0.05 was used to differentiate means according to Snedecor and Cochran (1980).

\section{RESULTS AND DISCUSSION}

All discussed data of this study are the mean values of the two seasons because of the similarity of results trend in the two seasons.

\section{Vegetative growth traits:}

Plant height, branches number plant ${ }^{-1}$ and shoot dry weight plant $^{-1}$ presented in Table (3) were significantly increased by $24.09 \%, 57.50 \%$ and $60.19 \%$, respectively when seeds treated with calcium paste then, sown in beds fecundated with ground sunflower heads as compared with these parameters when seeds sown in their beds without any treatments for both seeds and their beds alike. These improving effects on studied vegetative growth parameters may be due to that $\mathrm{Ca}^{2+}$ in calcium paste reduced the harmful effects of salinity on seeds and consequently positively reflected on germination and emergence percentages. In addition, it has been explained in a way that root hairs of plants were able to maintain higher levels of 
membrane-associated $\mathrm{Ca}^{2+}$ when exposed to high concentrations of $\mathrm{NaCl}$ (Cramer et al., 1985). In fact, it is essential to have $\mathrm{Ca}^{+}$of selective permeability, i.e. membrane integrity (Poovaiah and Reddy, 1993). This helped in improving vegetative growth traits through improving plant growth. Furthermore, ground sunflower heads found in seed-beds are able to maintenance of water (Table 2) in rizhosphere and consequently dilution of salinity around roots. Regarding results of this study, increasing macro-and microelements (N, P, K, Ca, Fe, Mn and $\mathrm{Zn}$ ) as shown in Tables (8-10) and the decrease in $\mathrm{Na}^{+}$(Table 9) induced by seed treatment with calcium paste and fecundating seed-beds with ground sunflower heads led to the significant increase in vegetative growth traits.

Table (3): Effect of calcium paste treated-or untreated-seeds sown in beds fecundated or non-fecundated with ground sunflower heads and foliar application with ascorbic acid on some growth traits of pea plants grown under reclaimed soil conditions during 2007/2008 and 2008/2009 seasons.

\begin{tabular}{|c|c|c|c|c|c|c|c|c|c|}
\hline \multirow{3}{*}{$\begin{array}{l}\text { Ascorbic acid } \\
\left(\mathrm{mgL}^{-1}\right)\end{array}$} & \multicolumn{2}{|c|}{$2007 / 2008$} & \multirow{2}{*}{$\begin{array}{c}\text { Mean } \\
\text { (B) }\end{array}$} & \multicolumn{2}{|c|}{$2008 / 2009$} & \multirow{2}{*}{$\begin{array}{l}\text { Mean } \\
\text { (B) }\end{array}$} & \multicolumn{2}{|c|}{$\begin{array}{c}\text { Mean of the two } \\
\text { seasons }\end{array}$} & \multirow{2}{*}{$\begin{array}{c}\text { Mean } \\
\text { (B) }\end{array}$} \\
\hline & $-\mathrm{Ca}^{+2}$ & $+\mathrm{Ca}^{+2}$ & & $-\mathbf{C a}^{+2}$ & $+\mathrm{Ca}^{+2}$ & & $-\mathbf{C a}^{+2}$ & $+\mathrm{Ca}^{+2}$ & \\
\hline & \multicolumn{9}{|c|}{ Plant height (cm) } \\
\hline 0 & 29.5 & 39.2 & 34.4 & 30.1 & 39.0 & 34.6 & 29.8 & 39.1 & 34.5 \\
\hline 100 & 34.1 & 45.3 & 39.7 & 34.0 & 46.2 & 40.1 & 34.1 & 45.8 & 40.0 \\
\hline 200 & 40.8 & 50.4 & 45.6 & 38.7 & 50.8 & 44.8 & 39.8 & 50.6 & 45.2 \\
\hline 300 & 51.6 & 59.2 & 55.4 & 50.3 & 60.4 & 55.4 & 51.0 & 59.8 & 55.4 \\
\hline 400 & 51.4 & 58.8 & 55.1 & 50.2 & 60.4 & 55.3 & 50.8 & 59.6 & 55.2 \\
\hline Mean (A) & 41.5 & 50.6 & & 40.7 & 51.4 & & 41.1 & 51.0 & \\
\hline$L^{L_{S}} D_{0.05} \quad A$ & \multicolumn{3}{|c|}{2.9} & \multicolumn{3}{|c|}{2.5} & \multicolumn{3}{|c|}{2.7} \\
\hline B & \multicolumn{3}{|c|}{4.4} & \multicolumn{3}{|c|}{3.9} & \multicolumn{3}{|c|}{4.2} \\
\hline AxB & \multicolumn{3}{|c|}{5.3} & \multicolumn{3}{|c|}{4.8} & \multicolumn{3}{|c|}{5.1} \\
\hline \multicolumn{10}{|c|}{ No. of branches plant ${ }^{-1}$} \\
\hline 0 & 1.12 & 2.00 & 1.56 & 1.20 & 2.00 & 1.60 & 1.16 & 2.00 & 1.58 \\
\hline 100 & 1.29 & 2.28 & 1.79 & 1.38 & 2.31 & 1.85 & 1.34 & 2.30 & 1.82 \\
\hline 200 & 1.50 & 2.56 & 2.03 & 1.52 & 2.60 & 2.06 & 1.51 & 2.58 & 2.05 \\
\hline 300 & 2.02 & 2.82 & 2.42 & 2.00 & 2.90 & 2.45 & 2.01 & 2.86 & 2.44 \\
\hline 400 & 2.00 & 2.81 & 2.41 & 2.00 & 2.90 & 2.45 & 2.00 & 2.86 & 2.43 \\
\hline Mean (A) & 1.59 & 2.49 & & 1.62 & 2.54 & & 1.60 & 2.52 & \\
\hline$L^{L^{2} D_{0.05}} \mathrm{~A}$ & \multicolumn{3}{|c|}{0.14} & \multicolumn{3}{|c|}{0.12} & \multicolumn{3}{|c|}{0.13} \\
\hline B & \multirow{2}{*}{\multicolumn{3}{|c|}{$\begin{array}{l}0.21 \\
0.27\end{array}$}} & \multirow{2}{*}{\multicolumn{3}{|c|}{$\begin{array}{l}0.19 \\
0.25\end{array}$}} & \multicolumn{3}{|c|}{0.20} \\
\hline AxB & & & & & & & & 0.26 & \\
\hline \multicolumn{10}{|c|}{ Shoot of dry weight plant ${ }^{-1}(\mathrm{~g})$} \\
\hline 0 & 6.09 & 10.21 & 8.15 & 6.20 & 10.03 & 8.12 & 6.15 & 10.12 & 8.14 \\
\hline 100 & 7.01 & 11.96 & 9.49 & 7.14 & 11.84 & 9.49 & 7.08 & 11.90 & 9.49 \\
\hline 200 & 8.18 & 13.80 & 10.99 & 8.12 & 13.69 & 10.91 & 8.15 & 13.75 & 10.95 \\
\hline 300 & 10.16 & 15.12 & 12.64 & 9.98 & 15.52 & 12.75 & 10.07 & 15.32 & 12.70 \\
\hline 400 & 10.09 & 15.10 & 12.60 & 9.95 & 15.50 & 12.73 & 10.02 & 15.30 & 12.66 \\
\hline Mean (A) & 8.31 & 13.24 & & 8.28 & 13.32 & & 8.29 & 13.28 & \\
\hline \begin{tabular}{|ll}
$\mathrm{LSD}_{0.05}$ & $\mathrm{~A}$ \\
\end{tabular} & \multicolumn{3}{|c|}{0.64} & \multicolumn{3}{|c|}{0.60} & \multicolumn{3}{|c|}{0.62} \\
\hline B & & 0.96 & & & 0.89 & & & 0.93 & \\
\hline AxB & & 1.28 & & & 1.18 & & & 1.23 & \\
\hline
\end{tabular}

$+\mathrm{Ca}=$ Seed covered with calcium paste and sown in beds fecundated with ground sunflower heads.

-Ca = Seed without calcium paste and sown in beds free from ground sunflower heads. 
All sub-main treatments; ascorbic acid at the rates of $0,100,200,300$ and $400 \mathrm{mgL}^{-1}$ (Table 3) reveal a gradual significant increase in all studied vegetative growth traits, i.e. plant height, branches number plant ${ }^{-1}$ and shoot dry weight plant $^{-1}$ was observed as a result in raising ascorbic acid rate gradually applied up to $300 \mathrm{mgL}^{-1}$. The rate of $400 \mathrm{mgL}^{-1}$ ascorbic acid proved to slightly decreased the mentioned parameters as compared with the rate of $300 \mathrm{mgL}^{-1}$. The maximum results were recorded with ascorbic acid foliar application at the rate of $300 \mathrm{mgL}^{-1}$ which surpassed the results of water foliar spray (the rate of zero $\mathrm{mgL}^{-1}$ ascorbic acid) by $60.58 \%, 54.43 \%$ and $56.02 \%$ for plant height, branches number plant ${ }^{-1}$ and shoot dry weight plant ${ }^{1}$, respectively. These results indicate that the most pronounced counteracted effects of studied soil salinity (Ca. $5000 \mathrm{ppm}$ ) on vegetative growth traits under study were overcomed by the exogenous application of ascorbic acid which led to the increase in endogenous level of this substance (Table 6) and consequently led to protect plant cells including protect the photosynthetic apparatus by scavenging reactive oxygen species (Zhang and Schmidt, 2000 ) thus, vigorous plant growth will be obtained under salinity stress. In this connection, Prusky (1988) and Elade (1992) stated a positive action for antioxidants especially, ascorbic acid on growth and attributed this finding to their effects on counteracting drought, salinity and diseases stresses and protecting plant cells against free radicals that responsible for plant senescence as well as to their auxinic action and consequently enhancing growth characters. In addition, ascorbic acid might regulate cell wall expansion, cell division and cell elongation through its action in cell vacuolarization (Arrigoni, 1994; Navas and Gomez-Diaz, 1995 and CordobaPedregosa et al., 1996), improves the nutritional status (Tables 8, 9 and 10) and absorbing phenolic compounds which lead to save the growing tissues from toxic effects of the oxidized phenols (Gupta et al., 1980) and/or enhances the biosynthesis of soluble sugars (Table 7) and carbohydrates (Rady, 2006). These findings are in coincidence with those obtained by Ahmed et al. (1998); Ragab (2002); Ahmed et al. (2003); Mostafa (2004) and Rady (2006).

The effect of the interactions between the main treatments (untreated seed sown in non-fecundated beds or treated seeds with calcium paste and sown in beds fecundated with ground sunflower heads) and sub-main treatments (ascorbic acid foliar application) was significant. The highest values of plant height, branches number plant ${ }^{-1}$ and shoot dry weight plant ${ }^{-1}$ were recorded from the treatment $300 \mathrm{mgL}^{-1}$ ascorbic acid under seed treatment with calcium paste plus seed-beds fecundated with ground sunflower heads which recorded $52.94 \%, 43.00 \%$ and $51.38 \%$ increases for plant height, branches number plant ${ }^{-1}$ and shoot dry weight plant ${ }^{-1}$, respectively above the treatment zero $\mathrm{mgL}^{-1}$ ascorbic acid under seed treatment with calcium paste plus seed-beds fecundated with ground sunflower heads and recorded $100.67 \%, 146.55 \%$ and $149.11 \%$ increases above the treatment zero $\mathrm{mgL}^{-1}$ ascorbic acid under untreated seeds and their beds. The superiority of the treatment having the highest values might come from improving the nutritional status of plants of this treatment (Tables 8-10), the shortage of $\mathrm{Na}^{+}$(Table 9) and the increase in total soluble sugars 
and proline (Table 7) saving more osmotic solutes which enable plant cells to maintain more water against salinity.

\section{Green yield and its components:}

Data in Tables (4 and 5) indicate that all green yield and its components under study, i.e. No. of green pods plant $^{-1}$, pod weight, total green pods yield feddan ${ }^{-1}$, No. of green seeds pod $^{-1}, 100$-seed weight and green seeds yield feddan ${ }^{-1}$ were significantly increased as a result of calcium paste-treated seeds sown in ground sunflower heads-fecundated beds which recorded $76.26 \%, 33.43 \%, 134.85 \%, 30.72 \%, 30.97 \%$ and $176.24 \%$ for above mentioned green yield and its components, respectively as compared with calcium paste-untreated seeds sown in ground sunflower heads-nonfecundated beds.

Table (4): Effect of calcium paste treated-or untreated-seeds sown in beds fecundated or non-fecundated with ground sunflower heads and foliar application with ascorbic acid on green yield and some of its components of pea plants grown under reclaimed soil conditions during 2007/2008 and 2008/2009 seasons.

\begin{tabular}{|c|c|c|c|c|c|c|c|c|c|}
\hline \multirow{3}{*}{$\begin{array}{l}\text { Ascorbic acid } \\
\left(\mathrm{mgL}^{-1}\right)\end{array}$} & \multicolumn{2}{|c|}{$2007 / 2008$} & \multirow{2}{*}{$\begin{array}{l}\text { Mean } \\
\text { (B) }\end{array}$} & \multicolumn{2}{|c|}{$2008 / 2009$} & \multirow{2}{*}{$\begin{array}{l}\text { Mean } \\
\text { (B) }\end{array}$} & \multicolumn{2}{|c|}{$\begin{array}{l}\text { Mean of the } \\
\text { two seasons }\end{array}$} & \multirow{2}{*}{$\begin{array}{l}\text { Mean } \\
\text { (B) }\end{array}$} \\
\hline & $-\mathrm{Ca}^{+2}$ & $+\mathrm{Ca}^{+2}$ & & $-\mathrm{Ca}^{+2}$ & $+\mathrm{Ca}^{+2}$ & & $-\mathrm{Ca}^{+2}$ & $+\mathrm{Ca}^{+2}$ & \\
\hline & \multicolumn{9}{|c|}{ No. of green pods plant ${ }^{-1}$} \\
\hline 0 & 5.38 & 8.10 & 6.74 & 5.41 & 8.39 & 6.90 & 5.40 & 8.24 & 6.82 \\
\hline 100 & 5.49 & 8.54 & 7.02 & 5.54 & 9.14 & 7.34 & 5.52 & 8.84 & 7.18 \\
\hline 200 & 5.81 & 10.68 & 8.24 & 5.98 & 10.92 & 8.45 & 5.90 & 10.80 & 8.35 \\
\hline 300 & 7.40 & 14.21 & 10.81 & 7.56 & 13.97 & 10.77 & 7.48 & 14.09 & 10.79 \\
\hline 400 & 7.36 & 14.29 & 10.83 & 7.60 & 13.90 & 10.75 & 7.48 & 14.10 & 10.79 \\
\hline Mean (A) & 6.29 & 11.16 & & 6.42 & 11.26 & & 6.36 & 11.21 & \\
\hline $\mathrm{LSD}_{0.05} \mathrm{~A}$ & \multicolumn{3}{|c|}{0.21} & \multicolumn{3}{|c|}{0.24} & \multicolumn{3}{|c|}{0.22} \\
\hline B & \multicolumn{3}{|c|}{0.30} & \multicolumn{3}{|c|}{0.36} & \multicolumn{3}{|c|}{0.33} \\
\hline AxB & \multicolumn{3}{|c|}{0.36} & \multicolumn{3}{|c|}{0.42} & \multicolumn{3}{|c|}{0.39} \\
\hline \multicolumn{10}{|c|}{ Pod weight (g) } \\
\hline 0 & 2.61 & 3.98 & 3.30 & 2.58 & 4.05 & 3.32 & 2.60 & 4.02 & 3.31 \\
\hline 100 & 2.98 & 4.11 & 3.55 & 2.89 & 4.19 & 3.54 & 2.94 & 4.15 & 3.55 \\
\hline 200 & 3.24 & 4.46 & 3.85 & 3.18 & 4.38 & 3.78 & 3.21 & 4.42 & 3.82 \\
\hline 300 & 4.00 & 5.10 & 4.55 & 4.03 & 4.91 & 4.47 & 4.02 & 5.01 & 4.52 \\
\hline 400 & 4.01 & 5.05 & 4.53 & 3.98 & 4.97 & 4.48 & 4.00 & 5.01 & 4.51 \\
\hline Mean (A) & 3.37 & 4.54 & & 3.33 & 4.50 & & 3.35 & 4.52 & \\
\hline \begin{tabular}{|ll}
$\mathrm{LSD}_{0.05}$ & $\mathrm{~A}$ \\
\end{tabular} & \multicolumn{3}{|c|}{0.18} & \multicolumn{3}{|c|}{0.14} & \multicolumn{3}{|c|}{0.16} \\
\hline B & \multicolumn{3}{|c|}{0.27} & \multicolumn{3}{|c|}{0.21} & \multicolumn{3}{|c|}{0.24} \\
\hline AxB & & 0.33 & & & 0.27 & & & 0.30 & \\
\hline \multicolumn{10}{|c|}{ Total green pods yield feddan ${ }^{-1}$ (ton) } \\
\hline 0 & 0.42 & 0.97 & 0.70 & 0.42 & 1.02 & 0.72 & 0.42 & 1.00 & 0.71 \\
\hline 100 & 0.49 & 1.05 & 0.77 & 0.48 & 1.15 & 0.82 & 0.49 & 1.10 & 0.80 \\
\hline 200 & 0.56 & 1.43 & 1.00 & 0.57 & 1.43 & 1.00 & 0.57 & 1.43 & 1.00 \\
\hline 300 & 0.89 & 2.15 & 1.52 & 0.90 & 2.08 & 1.49 & 0.90 & 2.12 & 1.51 \\
\hline 400 & 0.88 & 2.19 & 1.54 & 0.92 & 2.05 & 1.49 & 0.90 & 2.12 & 1.51 \\
\hline Mean (A) & 0.65 & 1.56 & & 0.66 & 1.55 & & 0.66 & 1.55 & \\
\hline \begin{tabular}{|ll}
$\mathrm{LSD}_{0.05}$ & $\mathrm{~A}$
\end{tabular} & \multicolumn{3}{|c|}{0.07} & \multicolumn{3}{|c|}{0.05} & \multicolumn{3}{|c|}{0.06} \\
\hline B & & 0.09 & & & 0.07 & & & 0.08 & \\
\hline AxB & & 0.12 & & & 0.08 & & & 0.10 & \\
\hline
\end{tabular}

$+\mathrm{Ca}=$ Seed covered with calcium paste and sown in beds fecundated with ground sunflower heads.

-Ca = Seed without calcium paste and sown in beds free from ground sunflower heads. 
Table (5): Effect of calcium paste treated-or untreated-seeds sown in beds fecundated or non-fecundated with ground sunflower heads and foliar application with ascorbic acid on green seed yield and some of its components of pea plants grown under reclaimed soil conditions during 2007/2008 and 2008/2009 seasons.

\begin{tabular}{|c|c|c|c|c|c|c|c|c|c|}
\hline \multirow{3}{*}{$\begin{array}{l}\text { Ascorbic acid } \\
\left(\mathrm{mgL}^{-1}\right)\end{array}$} & \multicolumn{2}{|c|}{ 2007/2008 } & \multirow{2}{*}{$\begin{array}{l}\text { Mean } \\
(\mathrm{B})\end{array}$} & \multicolumn{2}{|c|}{$2008 / 2009$} & \multirow{2}{*}{$\begin{array}{l}\text { Mean } \\
\text { (B) }\end{array}$} & \multicolumn{2}{|c|}{$\begin{array}{c}\text { Mean of the two } \\
\text { seasons }\end{array}$} & \multirow{2}{*}{$\begin{array}{l}\text { Mean } \\
\text { (B) }\end{array}$} \\
\hline & $-\mathrm{Ca}^{+2}$ & $+\mathrm{Ca}^{+2}$ & & $-\mathrm{Ca}^{+2}$ & $+\mathrm{Ca}^{+2}$ & & $-\mathrm{Ca}^{+2}$ & $+\mathrm{Ca}^{+2}$ & \\
\hline & \multicolumn{9}{|c|}{ No. of green seeds pod ${ }^{-1}$} \\
\hline 0 & 4.08 & 8.62 & 6.35 & 3.99 & 8.54 & 6.27 & 4.04 & 8.58 & 6.31 \\
\hline 100 & 5.16 & 8.96 & 7.06 & 5.07 & 9.00 & 7.04 & 5.12 & 8.98 & 7.05 \\
\hline 200 & 8.02 & 9.58 & 8.80 & 7.88 & 9.62 & 8.75 & 7.95 & 9.60 & 8.78 \\
\hline 300 & 9.61 & 10.12 & 9.87 & 9.58 & 10.20 & 9.89 & 9.60 & 10.16 & 9.88 \\
\hline 400 & 9.54 & 10.09 & 9.82 & 9.61 & 10.16 & 9.89 & 9.58 & 10.13 & 9.86 \\
\hline Mean (A) & 7.28 & 9.47 & & 7.23 & 9.50 & & 7.26 & 9.49 & \\
\hline \begin{tabular}{|ll}
$\mathbf{L S D}_{0.05}$ & $\mathrm{~A}$ \\
\end{tabular} & \multicolumn{3}{|c|}{0.48} & \multicolumn{3}{|c|}{0.45} & \multicolumn{3}{|c|}{0.47} \\
\hline B & \multicolumn{3}{|c|}{0.71} & \multicolumn{3}{|c|}{0.66} & \multicolumn{3}{|c|}{0.68} \\
\hline AxB & \multicolumn{3}{|c|}{0.90} & \multicolumn{3}{|c|}{0.86} & \multicolumn{3}{|c|}{0.88} \\
\hline \multicolumn{10}{|c|}{ 100-seed weight (g) } \\
\hline 0 & 10.93 & 19.54 & 15.24 & 11.08 & 20.12 & 15.60 & 11.01 & 19.83 & 15.42 \\
\hline 100 & 14.08 & 19.78 & 16.93 & 13.94 & 21.14 & 17.54 & 14.01 & 20.46 & 17.24 \\
\hline 200 & 18.50 & 20.63 & 19.57 & 18.60 & 21.90 & 20.25 & 18.55 & 21.27 & 19.91 \\
\hline 300 & 20.14 & 23.92 & 22.03 & 20.21 & 24.51 & 22.36 & 20.18 & 24.22 & 22.20 \\
\hline 400 & 20.21 & 23.88 & 22.05 & 20.18 & 24.46 & 22.32 & 20.20 & 24.17 & 22.19 \\
\hline Mean (A) & 16.77 & 21.55 & & 16.80 & 22.43 & & 16.79 & 21.99 & \\
\hline $\begin{array}{|ll|}\text { LSD }_{0.05} & A \\
\end{array}$ & & 0.96 & & & 1.20 & & & 1.08 & \\
\hline B & & 1.46 & & & 1.74 & & & 1.60 & \\
\hline AxB & & 1.78 & & & 2.14 & & & 1.96 & \\
\hline \multicolumn{10}{|c|}{ Green seeds yield feddan $^{-1}(\mathrm{~kg})$} \\
\hline 0 & 71.9 & 409.3 & 240.6 & 71.7 & 432.4 & 252.1 & 71.8 & 420.9 & 246.4 \\
\hline 100 & 119.6 & 454.0 & 286.8 & 117.4 & 521.6 & 319.5 & 118.5 & 487.8 & 303.2 \\
\hline 200 & 258.6 & 633.2 & 445.9 & 262.9 & 690.1 & 476.5 & 260.8 & 661.7 & 461.3 \\
\hline 300 & 429.6 & 1031.9 & 730.8 & 439.1 & 1047.7 & 743.4 & 434.4 & 1039.8 & 737.1 \\
\hline 400 & 425.7 & 1032.9 & 729.3 & 442.1 & 1036.3 & 739.2 & 433.9 & 1034.6 & 734.3 \\
\hline Mean (A) & 261.1 & 712.3 & & 266.6 & 745.6 & & 263.9 & 729.0 & \\
\hline $\begin{array}{|ll|}\mathbf{L S D}_{0.05} & \mathrm{~A} \\
\end{array}$ & \multicolumn{3}{|c|}{34.2} & \multicolumn{3}{|c|}{32.9} & \multicolumn{3}{|c|}{33.6} \\
\hline B & \multicolumn{3}{|c|}{51.4} & \multicolumn{3}{|c|}{49.8} & \multicolumn{3}{|c|}{50.6} \\
\hline AxB & \multicolumn{3}{|c|}{62.6} & \multicolumn{3}{|c|}{60.2} & \multicolumn{3}{|c|}{61.4} \\
\hline
\end{tabular}

$+\mathrm{Ca}=$ Seed covered with calcium paste and sown in beds fecundated with ground sunflower heads.

-Ca = Seed without calcium paste and sown in beds free from ground sunflower heads.

The improving in green yield and its components may be due to the existence of $\mathrm{Ca}^{++}$to take the place of $\mathrm{Na}^{+}$in the rizhosphere and in plant shoots as shown in Table (9) in this study which may be positively reflected on plant growth (Table 3) and plant pigments (Table 6) as well as macro- and microelements (Tables 8-10) and other components; proline, total soluble sugars, total phenols (Table 7) as osmotic substances and consequently increase yield and its components. Moreover, the better water retention capacity of ground sunflower heads (Table 2) enable rizhosphere to retain water to counteract the harmful effect of salinity. 
Table (6): Effect of calcium paste treated-or untreated-seeds sown in beds fecundated or non-fecundated with ground sunflower heads and foliar application with ascorbic acid on some leaf pigments and shoot ascorbic acid of pea plants grown under reclaimed soil conditions during 2007/2008 and 2008/2009 seasons.

\begin{tabular}{|c|c|c|c|c|c|c|c|c|c|}
\hline \multirow{3}{*}{$\begin{array}{l}\text { Ascorbic acid } \\
\left(\mathrm{mgL}^{-1}\right)\end{array}$} & \multicolumn{2}{|c|}{ 2007/2008 } & \multirow{2}{*}{$\begin{array}{l}\text { Mean } \\
\text { (B) }\end{array}$} & \multicolumn{2}{|c|}{$2008 / 2009$} & \multirow{2}{*}{$\begin{array}{l}\text { Mean } \\
\text { (B) }\end{array}$} & \multicolumn{2}{|c|}{$\begin{array}{l}\text { Mean of the } \\
\text { two seasons }\end{array}$} & \multirow{2}{*}{$\begin{array}{c}\text { Mean } \\
(\mathrm{B})\end{array}$} \\
\hline & $-\mathrm{Ca}^{+2}$ & $+\mathrm{Ca}^{+2}$ & & $-\mathrm{Ca}^{+2}$ & $+\mathrm{Ca}^{+2}$ & & $-\mathrm{Ca}^{+2}$ & $+\mathrm{Ca}^{+2}$ & \\
\hline & \multicolumn{9}{|c|}{ Total chlorophyll (mg g ${ }^{-1}$ F.wt.) } \\
\hline 0 & 0.81 & 1.40 & 1.11 & 0.86 & 1.38 & 1.12 & 0.84 & 1.39 & 1.12 \\
\hline 100 & 0.86 & 1.51 & 1.19 & 0.92 & 1.47 & 1.20 & 0.89 & 1.49 & 1.19 \\
\hline 200 & 0.93 & 1.68 & 1.31 & 0.99 & 1.70 & 1.35 & 0.96 & 1.69 & 1.33 \\
\hline 300 & 1.18 & 1.92 & 1.55 & 1.21 & 1.94 & 1.58 & 1.20 & 1.93 & 1.57 \\
\hline 400 & 1.17 & 1.90 & 1.54 & 1.21 & 1.91 & 1.56 & 1.19 & 1.91 & 1.55 \\
\hline Mean (A) & 0.99 & 1.68 & & 1.04 & 1.68 & & 1.02 & 1.68 & \\
\hline \begin{tabular}{|ll}
$\mathbf{L S D}_{0.05}$ & $\mathrm{~A}$ \\
\end{tabular} & \multicolumn{3}{|c|}{0.06} & \multicolumn{3}{|c|}{0.07} & \multicolumn{3}{|c|}{0.07} \\
\hline B & \multicolumn{3}{|c|}{0.08} & \multicolumn{3}{|c|}{0.10} & \multicolumn{3}{|c|}{0.09} \\
\hline AxB & \multicolumn{3}{|c|}{0.10} & \multicolumn{3}{|c|}{0.12} & \multicolumn{3}{|c|}{0.11} \\
\hline \multicolumn{10}{|c|}{ Total carotenoids (mg g ${ }^{-1}$ F.wt.) } \\
\hline 0 & 0.34 & 0.40 & 0.37 & 0.33 & 0.43 & 0.38 & 0.34 & 0.42 & 0.38 \\
\hline 100 & 0.38 & 0.44 & 0.41 & 0.38 & 0.48 & 0.43 & 0.38 & 0.46 & 0.42 \\
\hline 200 & 0.44 & 0.53 & 0.49 & 0.42 & 0.56 & 0.49 & 0.43 & 0.55 & 0.49 \\
\hline 300 & 0.51 & 0.61 & 0.56 & 0.50 & 0.65 & 0.58 & 0.51 & 0.63 & 0.57 \\
\hline 400 & 0.51 & 0.60 & 0.56 & 0.48 & 0.64 & 0.56 & 0.50 & 0.62 & 0.56 \\
\hline Mean (A) & 0.44 & 0.52 & & 0.42 & 0.55 & & 0.43 & 0.54 & \\
\hline $\begin{array}{|ll|}\text { LSD }_{0.05} & \mathrm{~A} \\
\end{array}$ & & 0.03 & & & 0.03 & & & 0.03 & \\
\hline B & & 0.04 & & & 0.05 & & & 0.05 & \\
\hline AxB & & 0.06 & & & 0.06 & & & 0.06 & \\
\hline \multicolumn{10}{|c|}{ Ascorbic acid (mg g ${ }^{-1}$ F.wt.) } \\
\hline 0 & 0.78 & 1.58 & 1.18 & 0.72 & 1.49 & 1.11 & 0.75 & 1.54 & 1.15 \\
\hline 100 & 0.84 & 1.79 & 1.32 & 0.76 & 1.60 & 1.18 & 0.80 & 1.70 & 1.25 \\
\hline 200 & 0.89 & 2.10 & 1.50 & 0.84 & 1.81 & 1.33 & 0.87 & 1.96 & 1.42 \\
\hline 300 & 1.14 & 2.53 & 1.84 & 1.07 & 2.38 & 1.73 & 1.11 & 2.46 & 1.79 \\
\hline 400 & 1.15 & 2.49 & 1.82 & 1.05 & 2.39 & 1.72 & 1.10 & 2.44 & 1.77 \\
\hline Mean (A) & 0.95 & 2.10 & & 0.89 & 1.93 & & 0.93 & 2.02 & \\
\hline $\begin{array}{|ll|}\mathbf{L S D}_{0.05} & \mathrm{~A} \\
\end{array}$ & \multicolumn{3}{|c|}{0.08} & \multicolumn{3}{|c|}{0.07} & \multicolumn{3}{|c|}{0.08} \\
\hline B & \multicolumn{3}{|c|}{0.12} & \multicolumn{3}{|c|}{0.10} & \multicolumn{3}{|c|}{0.10} \\
\hline AxB & \multicolumn{3}{|c|}{0.15} & \multicolumn{3}{|c|}{0.12} & \multicolumn{3}{|c|}{0.13} \\
\hline
\end{tabular}

$+\mathrm{Ca}=$ Seed covered with calcium paste and sown in beds fecundated with ground sunflower heads.

-Ca = Seed without calcium paste and sown in beds free from ground sunflower heads.

Sub-main treatment of ascorbic acid foliar application at the rate 300 $\mathrm{mgL}^{-1}$ gave the highest significant increase for green yield and its components as compared with other ascorbic acid rates; 100, 200 and 400 $\mathrm{mgL}^{-1}$ except the two rates; 300 and $400 \mathrm{mgL}^{-1}$ in relation to No. of green pods plant $^{-1}$ and total green pods yield feddan ${ }^{-1}$. These increases which scored by $300 \mathrm{mgL}^{-1}$ ascorbic acid were $58.21 \%, 36.56 \%, 112.68 \%, 56.58 \%$, $43.97 \%$ and $199.15 \%$ for No. of green pods plant $^{-1}$, pod weight, total green pods yield feddan ${ }^{-1}$, No. of green seeds pod $^{-1}, 100$-seed weight and green seeds yield feddan ${ }^{-1}$,respectively as compared to the treatment of zero rate ascorbic acid. The improving effect of ascorbic acid on green yield and its 
components was mainly attributed to its positive action on enhancing growth traits (Table 3 ), photosynthetic pigments of plant leaves (Table 6), cellular solutes, i.e. free proline, soluble sugars and soluble phenols (Table 7) for sustenance of cells turgor leading to maintenance of metabolic activities in plants and plant nutritional status (Tables 8-10).

Table (7): Effect of calcium paste treated-or untreated-seeds sown in beds fecundated or non-fecundated with ground sunflower heads and foliar application with ascorbic acid on some photosynthates in shoots of pea plants grown under reclaimed soil conditions during 2007/2008 and 2008/2009 seasons.

\begin{tabular}{|c|c|c|c|c|c|c|c|c|c|}
\hline \multirow{3}{*}{$\begin{array}{l}\text { Ascorbic acid } \\
\left(\mathrm{mgL}^{-1}\right)\end{array}$} & \multicolumn{2}{|c|}{$2007 / 2008$} & \multirow{2}{*}{$\begin{array}{l}\text { Mean } \\
\text { (B) }\end{array}$} & \multicolumn{2}{|c|}{$2008 / 2009$} & \multirow{2}{*}{$\begin{array}{l}\text { Mean } \\
\text { (B) }\end{array}$} & \multicolumn{2}{|c|}{$\begin{array}{c}\text { Mean of the two } \\
\text { seasons }\end{array}$} & \multirow{2}{*}{$\begin{array}{l}\text { Mean } \\
(B)\end{array}$} \\
\hline & $-\mathbf{C a}^{+2}$ & $+\mathrm{Ca}^{+2}$ & & $-\mathrm{Ca}^{+2}$ & $+\mathrm{Ca}^{+2}$ & & $-\mathrm{Ca}^{+2}$ & $+\mathrm{Ca}^{+2}$ & \\
\hline & \multicolumn{9}{|c|}{ Free proline (mg g ${ }^{-1}$ D.wt.) } \\
\hline 0 & 0.18 & 0.23 & 0.21 & 0.16 & 0.19 & 0.18 & 0.17 & 0.21 & 0.19 \\
\hline 100 & 0.21 & 0.26 & 0.24 & 0.18 & 0.22 & 0.20 & 0.20 & 0.24 & 0.22 \\
\hline 200 & 0.24 & 0.30 & 0.27 & 0.21 & 0.27 & 0.24 & 0.23 & 0.29 & 0.26 \\
\hline 300 & 0.29 & 0.34 & 0.32 & 0.28 & 0.33 & 0.31 & 0.29 & 0.34 & 0.32 \\
\hline 400 & 0.29 & 0.34 & 0.32 & 0.28 & 0.32 & 0.30 & 0.29 & 0.33 & 0.31 \\
\hline Mean (A) & 0.24 & 0.29 & & 0.22 & 0.27 & & 0.24 & 0.28 & \\
\hline \begin{tabular}{|ll}
$\mathrm{LSD}_{0.05}$ & $\mathrm{~A}$ \\
\end{tabular} & \multicolumn{3}{|c|}{0.02} & \multicolumn{3}{|c|}{0.02} & \multicolumn{3}{|c|}{0.02} \\
\hline B & \multicolumn{3}{|c|}{0.03} & \multicolumn{3}{|c|}{0.02} & \multicolumn{3}{|c|}{0.03} \\
\hline AxB & & 0.03 & & & 0.03 & & & 0.03 & \\
\hline \multicolumn{10}{|c|}{ Total soluble sugars (mg g ${ }^{-1}$ D.wt.) } \\
\hline 0 & 21.06 & 25.18 & 23.12 & 20.01 & 23.16 & 21.59 & 20.54 & 24.17 & 22.36 \\
\hline 100 & 24.67 & 29.40 & 27.04 & 23.72 & 27.80 & 25.76 & 24.20 & 28.60 & 26.40 \\
\hline 200 & 28.84 & 32.96 & 30.90 & 27.66 & 30.98 & 29.32 & 28.25 & 31.97 & 30.11 \\
\hline 300 & 34.10 & 38.28 & 36.19 & 32.10 & 36.84 & 34.47 & 33.10 & 37.56 & 35.33 \\
\hline 400 & 34.10 & 38.10 & 36.10 & 32.00 & 36.80 & 34.40 & 33.05 & 37.45 & 35.25 \\
\hline Mean (A) & 28.55 & 32.78 & & 27.10 & 31.12 & & 27.83 & 31.95 & \\
\hline $\begin{array}{|ll|}\mathrm{LSD}_{0.05} & \mathrm{~A} \\
\end{array}$ & \multicolumn{3}{|c|}{1.63} & \multicolumn{3}{|c|}{1.72} & \multicolumn{3}{|c|}{1.68} \\
\hline B & \multicolumn{3}{|c|}{2.41} & \multicolumn{3}{|c|}{2.56} & \multicolumn{3}{|c|}{2.49} \\
\hline AxB & \multicolumn{3}{|c|}{2.96} & \multicolumn{3}{|c|}{3.04} & \multicolumn{3}{|c|}{3.00} \\
\hline \multicolumn{10}{|c|}{ Total phenols (mg g ${ }^{-1}$ F.wt.) } \\
\hline 0 & 0.87 & 1.02 & 0.95 & 0.89 & 1.05 & 0.97 & 0.88 & 1.04 & 0.96 \\
\hline 100 & 0.97 & 1.11 & 1.04 & 0.98 & 1.16 & 1.07 & 0.98 & 1.14 & 1.06 \\
\hline 200 & 1.15 & 1.30 & 1.23 & 1.14 & 1.29 & 1.22 & 1.15 & 1.30 & 1.23 \\
\hline 300 & 1.38 & 1.49 & 1.44 & 1.29 & 1.51 & 1.40 & 1.34 & 1.50 & 1.42 \\
\hline 400 & 1.38 & 1.48 & 1.43 & 1.27 & 1.51 & 1.39 & 1.33 & 1.50 & 1.42 \\
\hline Mean (A) & 1.15 & 1.28 & & 1.11 & 1.30 & & 1.14 & 1.30 & \\
\hline $\begin{array}{|ll|}\mathrm{LSD}_{0.05} & \mathrm{~A} \\
\end{array}$ & \multicolumn{3}{|c|}{0.05} & \multicolumn{3}{|c|}{0.04} & \multicolumn{3}{|c|}{0.05} \\
\hline B & & 0.08 & & & 0.06 & & & 0.07 & \\
\hline AxB & & 0.11 & & & 0.08 & & & 0.10 & \\
\hline
\end{tabular}

$+\mathrm{Ca}=$ Seed covered with calcium paste and sown in beds fecundated with ground sunflower heads.

-Ca = Seed without calcium paste and sown in beds free from ground sunflower heads.

In this respect, Al-Qubaie (2002) stated that ascorbic acid as an antioxidant compound has an auxinic action and also synergistic effect on the biosynthesis of carbohydrates and controlling the incidence of most fungi on plants makes them in vigour states and reflects on green pods and seeds 
yields. Besides, the induced effect of ascorbic acid as one of vitamins on growth and yield may be due to that vitamins are recognized to be coenzymes involved in specific biochemical reactions in plants such as oxidative and non-oxidative decarboxylations (Robinson, 1973). The results regarding the beneficial effect of ascorbic acid on green yields of pods and seeds are confirmed with those reported by Ahmed et al. (2002) and (2003), Mostafa (2004) and Rady (2006).

As for the interaction between treated or untreated seeds with calcium paste sown in fecundated or non-fecundated beds with ground sunflower heads and ascorbic acid foliar application at various rates, data presented in Tables (4 and 5) represent that the highest significant increases were obtained from the application of $300 \mathrm{mgL}^{-1}$ ascorbic acid combined with treated seeds and fecundated seed-beds with calcium paste and ground sunflower heads, orderly for all tested green yield and its components. These increases obtained from this combined treatment scored at $71.00 \%, 24.63 \%$, $122.00 \%, 18.41 \%, 22.14 \%$ and $147.04 \%$, and

$160.93 \%, 92.69 \%, 404.76 \%, 151.49 \%, 119.98 \%$ and $1348.19 \%$ as compared to zero $\mathrm{mgL}^{-1}$ ascorbic acid under calcium paste-treated seeds sown in ground sunflower heads-fecundated beds and zero $\mathrm{mgL}^{-1}$ ascorbic acid under untreated seeds with calcium paste sown in non-fecundated beds with ground sunflower heads, respectively for No. of green pods plant ${ }^{-1}$, pod weight, total green pods yield feddan ${ }^{-1}$, No. of green seeds pod ${ }^{-1}, 100$-seed weight and green seeds yield feddan ${ }^{-1}$, orderly. This favourable production may be due to the positive combined effect of calcium and ground sunflower heads. The former has antagonistic effect to the harmful effects of $\mathrm{Na}^{+}$and the latter has high percentage of fibers (Table 2) have several physiological effects, depending upon the physical and chemical properties among them the ability of fibers to retain water and to bind organic compounds (Schneeman, 1986 and 1989) deluting salinity concentration and saving acidity effect in rizhosphere and consequently more solubility and absorption of nutrients by plant roots.

\section{Chemical constituents:}

a. Leaf pigments, ascorbic acid, proline, sugars and total phenols:

Data in Tables (6 and 7) reveal that total chlorophyll, total carotenoids, ascorbic acid, free proline, total soluble sugars and total soluble phenols in plant leaves or shoots were significantly increased in the order of $64.71 \%$, $25.58 \%, 117.20 \%, 16.67 \%, 14.80 \%$ and $14.04 \%$, respectively as a result of treating seeds with calcium paste plus fecundating seed-beds with ground sunflower heads as compared with the untreated seeds plus non-fecundated seed-beds. These pronounced increments may be due to the increase in ascorbic acid in plant shoots (Table 6) which has an auxinic actions and also synergistic effects on biosynthesis of sugars and carbohydrates (Al-Qubaie, 2002). The increase in phenols concentration in plant shoots (Table 7) resulting in the increase in ascorbic acid as an antioxidant thus, a linear relationship between ascorbic acid and total phenols concentration in plant shoots as represented in this study. Besides, the effective role of $\mathrm{Ca}^{++}$and ground sunflower heads in $\mathrm{Na}^{+}$driving away and more water retention in rizhosphere, respectively. 
Regarding the influence of ascorbic acid on total chlorophyll, total carotenoids, ascorbic acid, free proline, total soluble sugars and total soluble phenols concentrations in plant leaves or shoots, data shown in Tables (6 and 7) exhibit that all these parameters were gradually increased by significant quantities as a result of foliar application with ascorbic acid up to $300 \mathrm{mgL}^{-1}$ then neglictably decreased. The proportion of $40.18 \%, 50.00 \%$ $55.65 \%, 68.42 \%, 58.01 \%$ and $47.92 \%$ for total chlorophyll, total carotenoids, ascorbic acid, free proline, total soluble sugars and total soluble phenols, respectively were the increments of the best treatment; spraying plant shoots with ascorbic acid at the rate of $300 \mathrm{mgL}^{-1}$ as compared with the treatment free from ascorbic acid (tap water). The promotive effect of ascorbic acid on chlorophyll and carotenoids and the other components under study might be attributed to the enhancing effects of this antioxidant on the nutritional status of pea plants (Tables 8-10) since, $\mathrm{N}$ is one of the essential chlorophyll components. Besides, $\mathrm{Fe}$ and $\mathrm{Mn}$ are necessary for biosynthesis of chlorophyll and $\mathrm{Zn}$ is necessary for biosynthesis of tryptophan which is the precursor of auxin biosynthesis and consequently more biosynthesis of these components in the face of cells elongation. Furthermore, the role of ascorbic acid as an antioxidant, which directly involved in the regulation and protection of photosynthetic processes (Farago and Brunhold, 1994) could be led to the enhancing effect of ascorbic acid on pigments and the components under study. This treatment having the highest values of the constituents under study bestowed the tested plants the ability to satisfactorily overcome soil salinity under study in respect that their containing sufficient amount of soluble sugars, proline and soluble phenols (Table 7) which form sufficient cellular solutes able to sustenance of cell turgor leading to maintenance of metabolic activities in plant cells and/or protect plants against adverse condition, i.e. drought and salinity of such soil under study. The enhancing effect of ascorbic acid on tested soluble sugars might be attributed to its promotive effect also on studied pigments (Table 6) leading to the enhancement of photosynthesis and consequently, the increase in the photosynthates. The positive effects of ascorbic acid on above mentioned pigments and photosynthates obtained in this study are in agreement with those obtained by Tarraf et al. (1999); Ali (2002), Ahmed et al. (2003) and Rady (2006).

As regard to the results of the combination between treated or untreated seeds with calcium paste plus fecundated or non-fecundated seedbeds with ground sunflower heads exhibited in Tables ( 6 and 7 ) reveal that the combination between calcium paste treated-seeds sown in ground sunflower heads fecundated-beds and spraying plant shoots with ascorbic acid at $300 \mathrm{mgL}^{-1}$ preferable to all other combinations since granted the increases: $38.85 \%, 50.00 \%, 59.74 \%, 61.90 \%, 55.40 \%$ and $44.23 \%$ for total chlorophyll, total carotenoids, ascorbic acid, free proline, total soluble sugars and total soluble phenols, respectively as compared to calcium paste treatedseeds plus ground sunflower heads fecundated-seed-beds interacted with foliar spray with water (zero rate ascorbic acid), while when compared with the combined treatment between zero rate ascorbic acid and calcium paste untreated-seeds plus ground sunflower heads non-fecundated-seed-beds it 
scored increments at $129.76 \%, 85.29 \%, 228.00 \%, 100.00 \%, 82.86 \%$ and $70.45 \%$ for the same constituents, respectively. These significant increases scored as a result of the application with $\mathrm{Ca}^{++}$, ground sunflower heads as a fibrous source having high water holding capacity and ascorbic acid. The former may be reduced the harmful effects of salinity by its taking the place of $\mathrm{Na}^{+}$in rizhosphere and may on membranes in absorbing roots. The second; ground sunflower heads saves more water to overcome drought caused by salinity in the soil. The latter; ascorbic acid as one of antioxidants prevent enzyme inactivation, prevent the generation of more dangerous radicals and allow flexibility in the production of photosynthetic assimilatory power. Moreover, electron transfer to $\mathrm{O}_{2}$ prevented over reduction of electron transports chain, which reduced the risk of harmful back reaction

within the photosystem (Foyer et al., 1990). In addition, Elade (1992) and Farag (1996) proved that most antioxidants were responsible for accelerating the biosynthesis of various pigments and consequently more photosynthesis producing more quantities of photosynthates. Besides, Shahidi and Wanasundara (1992) stated that, phenolic antioxidants play important roles as free radical terminators and sometimes, as a metal chelators.

\section{B. Macro and micronutrients:}

It could be stated from data in Tables (8-10) that $\mathrm{N}, \mathrm{P}, \mathrm{K}, \mathrm{Ca}, \mathrm{Ca} / \mathrm{Na}$ ratio, $\mathrm{Fe}, \mathrm{Mn}$ and $\mathrm{Zn}$ were significantly increased with the treatment of calcium paste treated-seeds plus ground sunflower heads fecundated-seedbeds as compared with other treatment in which seeds and their beds were free from calcium paste and ground sunflower heads. These increases were recorded at $19.84 \%, 39.06 \%, 18.43 \%, 106.62 \%, 132.18 \%, 10.47 \%, 8.03 \%$ and $11.41 \%$ for $\mathrm{N}, \mathrm{P}, \mathrm{K}, \mathrm{Ca}$ and $\mathrm{Ca} / \mathrm{Na}$ ratio, $\mathrm{Fe}, \mathrm{Mn}$ and $\mathrm{Zn}$, respectively. On the other side, $\mathrm{Na}$ represents reversed behaviour, since decreased with increasing other elements at $13.17 \%$ in plant shoots. The shortage occurred in $\mathrm{Na}^{+}$and the increase in $\mathrm{Ca}^{++}$; the increase in $\mathrm{Ca} / \mathrm{Na}$ ratio under this study besides, the high water retention capacity and having some minerals such as $\mathrm{Ca}, \mathrm{Fe}$ and $\mathrm{Zn}$ as well as some vitamins (i.e. A and $\mathrm{E}$ ) of ground sunflower heads and wheat bran (Kahlan et al., 1986 and Klopfenstein, 1990) might be explained these results.

Data presented in Tables (8-10) reveal that regardless $\mathrm{Na}$ which behaved contrary case, all nutrients under studies i.e. $\mathrm{N}, \mathrm{P}, \mathrm{K}, \mathrm{Ca}, \mathrm{Ca} / \mathrm{Na}$ ratio, $\mathrm{Fe}, \mathrm{Mn}$ and $\mathrm{Zn}$ represented significant gradual increases with increasing ascorbic acid rate. The applied treatment of ascorbic acid at the rate of $300 \mathrm{mgL}^{-1}$ proved to be the best, and exhibited, in general, the most pronounced counteracted effect on soil salinity of this study. Such treatment surpassed the treatment of zero $\mathrm{mgL}^{-1}$ ascorbic acid (water) by $30.11 \%, 44.15 \%$, $36.63 \%, 27.82 \%, 50.00 \%, 23.38 \%, 22.18 \%$ and $22.71 \%$ for $\mathrm{N}, \mathrm{P}, \mathrm{K}, \mathrm{Ca}$, and $\mathrm{Ca} / \mathrm{Na}$ ratio, $\mathrm{Fe}, \mathrm{Mn}$ and $\mathrm{Zn}$, respectively. On the other hand, $\mathrm{Na}$ recorded shortage at $20.59 \%$ under the best treatment as compared with spraying water treatment. The beneficial effect of ascorbic acid on increasing tolerance of pea plants, especially at the rate of $300 \mathrm{mgL}^{-1}$ under studied soil salinity which reflected on improving vegetative growth traits (Table 3), photosynthates (Table 7), photosynthetic pigments and endogenous ascorbic acid (Table 6) and green yield of pods and seeds (Tables 4 and 5), surely 
reflected also on stimulating the nutritional status of plants. These results were supported by the results of Ahmed and Abd El-Hameed (2004) and Rady (2006) who reported that the effect of antioxidants, especially ascorbic acid on producing healthy plants leads to enhancing the plants to have a great ability for uptake of elements. Moreover, Gonzalez-Reyes et al. (1994) concluded that ascorbate free radical caused hyperpolarization of plasma membranes, and this energization could then facilitate transport processes across such membranes. Most of the previous results are consistent with those of Ali (2000); Ahmed et al. (2002) and Rady (2006).

Table (8): Effect of calcium paste treated-or untreated-seeds sown in beds fecundated or non-fecundated with ground sunflower heads and foliar application with ascorbic acid on some macronutrients in shoots of pea plants grown under reclaimed soil conditions during 2007/2008 and 2008/2009 seasons.

\begin{tabular}{|c|c|c|c|c|c|c|c|c|c|}
\hline \multirow{3}{*}{$\begin{array}{l}\text { Ascorbic acid } \\
\left(\mathrm{mgL}^{-1}\right)\end{array}$} & \multicolumn{2}{|c|}{$2007 / 2008$} & \multirow{2}{*}{$\begin{array}{c}\text { Mean } \\
\text { (B) }\end{array}$} & \multicolumn{2}{|c|}{$2008 / 2009$} & \multirow{2}{*}{$\begin{array}{c}\text { Mean } \\
\text { (B) }\end{array}$} & \multicolumn{2}{|c|}{$\begin{array}{c}\text { Mean of the two } \\
\text { seasons }\end{array}$} & \multirow[t]{2}{*}{$\begin{array}{l}\text { Mean } \\
\text { (B) }\end{array}$} \\
\hline & $-\mathrm{Ca}^{+2}$ & $+\mathrm{Ca}^{+2}$ & & $-\mathbf{C a}^{+2}$ & $+\mathrm{Ca}^{+2}$ & & \begin{tabular}{|c|}
$-\mathrm{Ca}^{+2}$ \\
\end{tabular} & $+\mathrm{Ca}^{+2}$ & \\
\hline & \multicolumn{9}{|c|}{$\mathbf{N}$ (mg g ${ }^{-1}$ D.wt.) } \\
\hline 0 & 21.36 & 25.10 & \begin{tabular}{|l|}
23.23 \\
\end{tabular} & 20.18 & 23.96 & 22.07 & 20.77 & 24.53 & 22.65 \\
\hline 100 & 22.49 & 27.08 & 24.79 & 21.20 & 25.44 & 23.32 & 21.85 & 26.26 & 24.06 \\
\hline 200 & 24.13 & 29.74 & \begin{tabular}{|l|}
26.94 \\
\end{tabular} & 22.84 & 27.18 & 25.01 & 23.49 & 28.46 & 25.98 \\
\hline 300 & 27.56 & 33.26 & 30.41 & 26.07 & 30.95 & 28.51 & 26.82 & 32.11 & 29.47 \\
\hline 400 & 27.40 & 33.30 & \begin{tabular}{|l|}
30.35 \\
\end{tabular} & 26.11 & 30.90 & 28.51 & 26.76 & 32.10 & 29.43 \\
\hline Mean (A) & 24.59 & 29.70 & & 23.28 & 27.69 & & 23.94 & 28.69 & \\
\hline $\begin{array}{|ll|}\text { LSD }_{0.05} & \mathrm{~A} \\
\end{array}$ & \multicolumn{3}{|c|}{1.49} & \multicolumn{3}{|c|}{1.56} & \multicolumn{3}{|c|}{1.53} \\
\hline B & \multicolumn{3}{|c|}{2.12} & \multicolumn{3}{|c|}{2.29} & \multicolumn{3}{|c|}{2.21} \\
\hline AxB & & 2.65 & & & 2.81 & & & 2.73 & \\
\hline \multicolumn{10}{|c|}{$\mathbf{P}$ (mg g ${ }^{-1}$ D.wt.) } \\
\hline 0 & 1.52 & 2.21 & 1.87 & 1.60 & 2.18 & 1.89 & 1.56 & 2.20 & 1.88 \\
\hline 100 & 1.61 & 2.33 & 1.97 & 1.68 & 2.30 & 1.99 & 1.65 & 2.32 & 1.99 \\
\hline 200 & 1.80 & 2.62 & 2.21 & 1.83 & 2.54 & 2.19 & 1.82 & 2.58 & 2.20 \\
\hline 300 & 2.30 & 3.12 & 2.71 & 2.24 & 3.17 & 2.71 & 2.27 & 3.15 & 2.71 \\
\hline 400 & 2.31 & 3.09 & 2.70 & 2.27 & 3.13 & 2.70 & 2.29 & 3.11 & 2.70 \\
\hline Mean (A) & 1.91 & 2.67 & & 1.92 & 2.66 & & 1.92 & 2.67 & \\
\hline $\begin{array}{|ll|}\text { LSD }_{0.05} & A \\
\end{array}$ & & 0.06 & & & 0.07 & & & 0.07 & \\
\hline B & & 0.09 & & & 0.10 & & & 0.10 & \\
\hline AxB & & 0.11 & & & 0.12 & & & 0.12 & \\
\hline \multicolumn{10}{|c|}{ (mg g ${ }^{-1}$ D.wt.) } \\
\hline 0 & 17.12 & 20.17 & 18.65 & 16.88 & 21.05 & 18.97 & 17.00 & 20.61 & 18.81 \\
\hline 100 & 18.20 & 20.96 & \begin{tabular}{|l|}
19.58 \\
\end{tabular} & 18.24 & 21.97 & 20.11 & 18.22 & 21.47 & 19.85 \\
\hline 200 & 20.14 & 23.66 & 21.90 & 19.86 & 23.40 & 21.63 & 20.00 & 23.53 & 21.77 \\
\hline 300 & 23.08 & 27.43 & 25.26 & 22.90 & 26.86 & 24.88 & 22.99 & 27.15 & 25.07 \\
\hline 400 & 23.10 & 27.38 & 25.24 & 22.86 & 26.80 & 24.83 & 22.98 & 27.09 & 25.04 \\
\hline $\operatorname{Mean}(\mathrm{A})$ & \begin{tabular}{|l|}
20.33 \\
\end{tabular} & 23.92 & & 20.15 & 24.02 & & 20.24 & 23.97 & \\
\hline $\begin{array}{|ll|}\text { LSD }_{0.05} & \mathrm{~A}\end{array}$ & & 0.72 & & & 0.68 & & & 0.70 & \\
\hline B & & 1.05 & & & 0.96 & & & 1.01 & \\
\hline AxB & & 1.24 & & & 1.20 & & & 1.22 & \\
\hline
\end{tabular}


Table (9): Effect of calcium paste treated-or untreated-seeds sown in beds fecundated or non-fecundated with ground sunflower heads and foliar application with ascorbic acid on $\mathrm{Ca}, \mathrm{Na}$ and $\mathrm{Ca} / \mathrm{Na}$ ratio in shoots of pea plants grown under reclaimed soil conditions during 2007/2008 and 2008/2009 seasons.

\begin{tabular}{|c|c|c|c|c|c|c|c|c|c|}
\hline \multirow{3}{*}{$\begin{array}{l}\text { Ascorbic acid } \\
\left(\mathrm{mgL}^{-1}\right)\end{array}$} & \multicolumn{2}{|c|}{$2007 / 2008$} & \multirow{2}{*}{$\begin{array}{l}\text { Mean } \\
\text { (B) }\end{array}$} & \multicolumn{2}{|c|}{$2008 / 2009$} & \multirow{2}{*}{$\begin{array}{l}\text { Mean } \\
\text { (B) }\end{array}$} & \multicolumn{2}{|c|}{$\begin{array}{l}\text { Mean of the } \\
\text { two seasons }\end{array}$} & \multirow{2}{*}{ Mean (B) } \\
\hline & $-\mathrm{Ca}^{+2}$ & $+\mathrm{Ca}^{+2}$ & & $-\mathrm{Ca}^{+2}$ & $+\mathrm{Ca}^{+2}$ & & $-\mathrm{Ca}^{+2}$ & $+\mathrm{Ca}^{+2}$ & \\
\hline & \multicolumn{9}{|c|}{ Ca (mg g ${ }^{-1}$ D.wt.) } \\
\hline 0 & 2.29 & 4.61 & 3.45 & 2.51 & 5.08 & 3.80 & 2.40 & 4.85 & 3.63 \\
\hline 100 & 2.41 & 4.90 & 3.66 & 2.68 & 5.31 & 4.00 & 2.55 & 5.11 & 3.83 \\
\hline 200 & 2.58 & 5.29 & 3.94 & 2.84 & 5.69 & 4.27 & 2.71 & 5.49 & 4.10 \\
\hline 300 & 2.81 & 6.34 & 4.58 & 3.09 & 6.31 & 4.70 & 2.95 & 6.33 & 4.64 \\
\hline 400 & 2.80 & 6.29 & 4.55 & 3.13 & 6.30 & 4.72 & 2.97 & 6.30 & 4.64 \\
\hline Mean (A) & 2.58 & 5.49 & & 2.85 & 5.74 & & 2.72 & 5.62 & \\
\hline \begin{tabular}{|ll}
$\mathbf{L S D}_{0.05}$ & $\mathrm{~A}$ \\
\end{tabular} & \multicolumn{3}{|c|}{0.12} & \multicolumn{3}{|c|}{0.13} & \multicolumn{3}{|c|}{0.13} \\
\hline B & \multicolumn{3}{|c|}{0.18} & \multicolumn{3}{|c|}{0.20} & \multicolumn{3}{|c|}{0.19} \\
\hline AxB & \multicolumn{3}{|c|}{0.22} & \multicolumn{3}{|c|}{0.24} & \multicolumn{3}{|c|}{0.23} \\
\hline \multicolumn{10}{|c|}{$\mathrm{Na}$ (mg g ${ }^{-1}$ D.wt.) } \\
\hline 0 & 3.61 & 2.96 & 3.29 & 3.50 & 3.01 & 3.26 & 3.56 & 2.99 & 3.28 \\
\hline 100 & 3.42 & 2.93 & 3.18 & 3.46 & 2.95 & 3.21 & 3.44 & 2.94 & 3.19 \\
\hline 200 & 3.19 & 2.87 & 3.03 & 3.28 & 2.90 & 3.09 & 3.24 & 2.89 & 3.07 \\
\hline 300 & 2.74 & 2.61 & 2.68 & 2.90 & 2.63 & 2.77 & 2.82 & 2.62 & 2.72 \\
\hline 400 & 2.73 & 2.60 & 2.67 & 2.92 & 2.65 & 2.79 & 2.83 & 2.63 & 2.73 \\
\hline Mean (A) & 3.14 & 2.79 & & 3.21 & 2.83 & & 3.18 & 2.81 & \\
\hline $\begin{array}{|ll|}\text { LSD }_{0.05} & \mathrm{~A} \\
\end{array}$ & & 0.08 & & & 0.08 & & & 0.08 & \\
\hline B & & 0.11 & & & 0.12 & & & 0.12 & \\
\hline AxB & & 0.13 & & & 0.14 & & & 0.14 & \\
\hline \multicolumn{10}{|c|}{$\mathrm{Ca} / \mathrm{Na}$ ratio } \\
\hline 0 & 0.63 & 1.58 & 1.11 & 0.72 & 1.69 & 1.21 & 0.68 & 1.64 & 1.16 \\
\hline 100 & 0.70 & 1.67 & 1.19 & 0.77 & 1.80 & 1.29 & 0.74 & 1.74 & 1.24 \\
\hline 200 & 0.81 & 1.84 & 1.33 & 0.87 & 1.96 & 1.42 & 0.84 & 1.90 & 1.37 \\
\hline 300 & 1.03 & 2.43 & 1.73 & 1.07 & 2.40 & 1.74 & 1.05 & 2.42 & 1.74 \\
\hline 400 & 1.03 & 2.42 & 1.73 & 1.07 & 2.38 & 1.73 & 1.05 & 2.40 & 1.73 \\
\hline Mean (A) & 0.84 & 1.99 & & 0.90 & 2.05 & & 0.87 & 2.02 & \\
\hline $\begin{array}{|ll|}\mathbf{L S D}_{0.05} & \mathrm{~A} \\
\end{array}$ & \multicolumn{3}{|c|}{0.05} & \multicolumn{3}{|c|}{0.06} & \multicolumn{3}{|c|}{0.06} \\
\hline B & \multicolumn{3}{|c|}{0.08} & \multicolumn{3}{|c|}{0.08} & \multicolumn{3}{|c|}{0.08} \\
\hline AxB & \multicolumn{3}{|c|}{0.10} & \multicolumn{3}{|c|}{0.10} & \multicolumn{3}{|c|}{0.10} \\
\hline
\end{tabular}

$+\mathrm{Ca}=$ Seed covered with calcium paste and sown in beds fecundated with ground sunflower heads.

- $\mathrm{Ca}=$ Seed without calcium paste and sown in beds free from ground sunflower heads.

Data presented in Tables (8-10) show that the best treatment by which plant shoots collected the highest amounts of nutrients except with the reverse regarding $\mathrm{Na}$, was treating seeds with calcium paste plus fecundating seed-beds with ground sunflower heads interacted with spraying plant shoots with $300 \mathrm{mgL}^{-1}$ ascorbic acid solution. This treatment scored $30.90 \%$, $43.18 \%, 31.73 \%, 30.52 \%, 47.56 \%, 24.21 \%, 22.33 \%$ and $20.75 \%$ for N, P, K, $\mathrm{Ca}, \mathrm{Ca} / \mathrm{Na}$ ratio, $\mathrm{Fe}, \mathrm{Mn}$ and $\mathrm{Zn}$, respectively when compared with the treatment in which seeds and their beds treated plus fecundated with calcium paste and ground sunflower heads interacted with plant shoots sprayed with water, orderly, while $\mathrm{Na}$ decreased down to $14.12 \%$. in addition, the same 
best treatment granted increases at $54.60 \%, 101.92 \%, 59.71 \%, 163.75 \%$, $255.88 \%, 35.64 \%, 32.09 \%$ and $37.02 \%$ for the same parameters, respectively as compared with the treatment under the interaction between calcium paste untreated-seeds plus ground sunflower heads non-fecundatedseed-beds and spraying plant foliage with water, whereas $\mathrm{Na}$ reduced at $35.88 \%$.

Table (10): Effect of calcium paste treated-or untreated-seeds sown in beds fecundated or non-fecundated with ground sunflower heads and foliar application with ascorbic acid on some micronutrients in shoots of pea plants grown under reclaimed soil conditions during 2007/2008 and 2008/2009 seasons.

\begin{tabular}{|c|c|c|c|c|c|c|c|c|c|}
\hline \multirow{3}{*}{$\begin{array}{l}\text { Ascorbic acid } \\
\left(\mathrm{mgL}^{-1}\right)\end{array}$} & \multicolumn{2}{|c|}{$2007 / 2008$} & \multirow{2}{*}{$\begin{array}{l}\text { Mean } \\
(B)\end{array}$} & \multicolumn{2}{|c|}{$2008 / 2009$} & \multirow{2}{*}{$\begin{array}{l}\text { Mean } \\
(\mathrm{B})\end{array}$} & \multicolumn{2}{|c|}{$\begin{array}{c}\text { Mean of the two } \\
\text { seasons }\end{array}$} & \multirow[t]{2}{*}{ Mean (B } \\
\hline & $-\mathrm{Ca}^{+2}$ & $+\mathrm{Ca}^{+2}$ & & $-\mathrm{Ca}^{+2}$ & $+\mathrm{Ca}^{+2}$ & & $-\mathrm{Ca}^{+2}$ & $+\mathrm{Ca}^{+2}$ & \\
\hline & \multicolumn{9}{|c|}{ Fe (ppm) } \\
\hline 0 & 420.6 & 461.4 & 441.0 & 431.2 & 468.8 & 450.0 & 425.9 & 465.1 & 445.6 \\
\hline 100 & 439.8 & 489.6 & 464.7 & 454.7 & 496.4 & 475.6 & 447.3 & 493.0 & 470.2 \\
\hline 200 & 464.9 & 518.9 & 491.9 & 478.3 & 531.0 & 504.7 & 471.6 & 525.0 & 498.3 \\
\hline 300 & 516.9 & 573.6 & 545.3 & 526.6 & 581.7 & 554.2 & 521.8 & 577.7 & 549.4 \\
\hline 400 & 518.3 & 571.2 & 544.8 & 524.8 & 582.9 & 553.9 & 521.6 & 577.1 & 549.8 \\
\hline Mean (A) & 472.1 & 522.9 & & 483.1 & 532.2 & & 477.6 & 527.6 & \\
\hline $\begin{array}{|ll|}\text { LSD }_{0.05} & \text { A }\end{array}$ & \multicolumn{3}{|c|}{16.3} & \multicolumn{3}{|c|}{15.8} & \multicolumn{3}{|c|}{16.1} \\
\hline B & \multicolumn{3}{|c|}{24.5} & \multicolumn{3}{|c|}{23.7} & \multicolumn{3}{|c|}{24.1} \\
\hline AxB & \multicolumn{3}{|c|}{29.8} & \multicolumn{3}{|c|}{30.2} & \multicolumn{3}{|c|}{30.0} \\
\hline \multicolumn{10}{|c|}{ Mn (ppm) } \\
\hline 0 & 250.0 & 271.6 & 260.8 & 246.7 & 264.7 & 255.7 & 248.4 & 268.2 & 258.3 \\
\hline 100 & 264.8 & 290.3 & 277.6 & 258.8 & 278.9 & 268.9 & 261.8 & 284.6 & 273.2 \\
\hline 200 & 281.7 & 304.9 & 293.3 & 274.6 & 295.1 & 284.9 & 278.2 & 300.0 & 289.1 \\
\hline 300 & 309.6 & 326.8 & 318.2 & 296.9 & 329.4 & 313.2 & 303.3 & 328.1 & 315.6 \\
\hline 400 & 310.0 & 324.3 & 317.2 & 296.0 & 326.9 & 311.5 & 303.0 & 325.6 & 314.3 \\
\hline Mean (A) & 283.2 & 303.6 & & 274.6 & 299.0 & & 278.9 & 301.3 & \\
\hline $\begin{array}{|ll|}\text { LSD }_{0.05} & A \\
\end{array}$ & \multicolumn{3}{|c|}{8.4} & \multicolumn{3}{|c|}{9.2} & \multicolumn{3}{|c|}{8.8} \\
\hline B & \multicolumn{3}{|c|}{12.6} & \multicolumn{3}{|c|}{13.8} & \multicolumn{3}{|c|}{13.2} \\
\hline$A \times B$ & \multicolumn{3}{|c|}{14.8} & \multicolumn{3}{|c|}{15.6} & \multicolumn{3}{|c|}{15.2} \\
\hline \multicolumn{10}{|c|}{ Zn (ppm) } \\
\hline 0 & 168.4 & 194.7 & 181.6 & 171.4 & 190.8 & 181.1 & 169.9 & 192.8 & 181.4 \\
\hline 100 & 176.3 & 203.9 & 190.1 & 180.6 & 198.9 & 189.8 & 178.5 & 201.4 & 190.0 \\
\hline 200 & 190.8 & 214.7 & 202.8 & 189.8 & 212.4 & 201.1 & 190.3 & 213.6 & 202.0 \\
\hline 300 & 219.3 & 233.6 & 226.5 & 205.4 & 231.9 & 218.7 & 212.4 & 232.8 & 222.6 \\
\hline 400 & 219.7 & 234.0 & 226.9 & 203.9 & 230.7 & 217.3 & 211.8 & 232.4 & 222.1 \\
\hline Mean (A) & 194.9 & 216.2 & & 190.2 & 212.9 & & 192.6 & 214.6 & \\
\hline $\begin{array}{|ll|}\mathbf{L S D}_{0.05} & \mathrm{~A} \\
\end{array}$ & & 5.8 & & & 5.4 & & & 5.6 & \\
\hline B & & 8.1 & & & 8.0 & & & 8.1 & \\
\hline AxB & & 10.0 & & & 9.8 & & & 9.9 & \\
\hline
\end{tabular}

$+\mathrm{Ca}=$ Seed covered with calcium paste and sown in beds fecundated with ground sunflower heads.

-Ca = Seed without calcium paste and sown in beds free from ground sunflower heads.

The increments which obtained from the above mentioned best treatment may be explaine with the increasing value of $\mathrm{Ca} / \mathrm{Na}$ ratio which reveal that $\mathrm{Ca}^{++}$to behave oneself when takes out $\mathrm{Na}^{+}$at significant amounts from 
rizhosphere and consequently from plant shoots (Table 9) which reflected on increasing nutrients in plants.

Besides, the water imbibing properties of ground sunflower heads and wheat bran fibers (Schneeman, 1986), and their ability to absorb water and/or organic compound (Mongeau and Brassard, 1982) support the rizhophere by water to face of drought and salinity, and acidity to facilitate more solubility and absorption of nutrients. As for ascorbic acid, Wise and Naylor (1987) stated that antioxidants such as ascorbate, glutathione and $\alpha$ tocopherol are directly correlated with the ability to defend plant cells against oxidative damage resulting from salinity stress and consequently producing healthy plants having a great ability for nutrients uptake.

\section{Chemical composition of green seeds:}

Starch, total soluble sugars and protein concentrations as shown in Table (11) were significantly affected under the application with $\mathrm{Ca}^{++}$and ground sunflower heads. This influence was positive for starch\% and protein $\%$ up to $12.00 \%$ and $18.12 \%$, respectively and negative for total soluble sugars $\%$ calculated at $9.88 \%$ under the treatment of calcium paste treated-seeds sown in ground sunflower heads fecundated-beds as compared with the treatment of untreating seeds with calcium paste plus nonfecundating seed-beds with ground sunflower heads. The improving effect of the best treatment of calcium paste treated-seeds sown in beds fecundated with ground sunflower heads on the nutritional status of plant shoots (Tables 8-10) reflected on photosynthetic pigments, growth characters and their photosynthates (Tables 3, 5 and 6) and thus reflected on green seeds yield, surely reflected on stored photosynthates in seeds. The reduction in total soluble sugars may be due to that soluble carbohydrates store in pea seeds in the form of starch.

A gradual significant increase was observed for starch\% and protein $\%$ and the contrary for total soluble sugars\% as a result of increasing the ascorbic acid rate from zero up to $300 \mathrm{mgL}^{-1}$ then, maintained at almost the same result with $400 \mathrm{mgL}^{-1}$ ascorbic acid solution. The best treatment; foliar application with $300 \mathrm{mgL}^{-1}$ ascorbic acid solution surpassed the treatment of water foliar application by $13.01 \%$ and $18.57 \%$ for starch $\%$ and protein $\%$, respectively while, reduced total soluble sugars $\%$ up to $17.79 \%$. The positive role of ascorbic acid on photosynthetic pigments (Table 6), photosynthates (Table 7) and nutritional status (Tables 8-10) which reflected on green seed yield, surely reflected on stored seed photosynthates.

The results presented in Table (11) show that the combined treatment of foliar application with ascorbic acid at the rate of $300 \mathrm{mgL}^{-1}$ under sown calcium paste treated-seeds in beds fecundated with ground sunflower heads granted the highest values at $12.93 \%$ and $20.76 \%$ for the percentage of starch and protein, respectively as compared with the combined treatment of zero rate of ascorbic acid solution under sown calcium paste treated-seeds in beds fecundated with ground sunflower heads, and at $25.81 \%$ and $38.82 \%$ for the same parameter, respectively as comparison with the combined treatment of foliar application with water under sown calcium paste untreated-seeds in beds non-fecundated with ground sunflower heads. The favourable positive effect of $\mathrm{Ca}^{++}$, ground sunflower heads and ascorbic 
acid on tested growth traits (Table 3), photosynthetic pigments (Table 6) nutritional and photosynthates status (Tables 7-10) which positively reflected on green seed yield (Table 5), surely reflected on stored materials in these seeds.

Table (11): Effect of calcium paste treated-or untreated-seeds sown in beds fecundated or non-fecundated with ground sunflower heads and foliar application with ascorbic acid on some stored components in green seeds of pea plants grown under reclaimed soil conditions during 2007/2008 and 2008/2009 seasons.

\begin{tabular}{|c|c|c|c|c|c|c|c|c|c|}
\hline \multirow{3}{*}{$\begin{array}{l}\text { Ascorbic acid } \\
\left(\mathrm{mgL}^{-1}\right)\end{array}$} & \multicolumn{2}{|c|}{$2007 / 2008$} & \multirow{2}{*}{$\begin{array}{c}\text { Mean } \\
\text { (B) }\end{array}$} & \multicolumn{2}{|c|}{$2008 / 2009$} & \multirow{2}{*}{$\begin{array}{c}\text { Mean } \\
\text { (B) }\end{array}$} & \multicolumn{2}{|c|}{$\begin{array}{c}\text { Mean of the two } \\
\text { seasons }\end{array}$} & \multirow{2}{*}{ Mean (B) } \\
\hline & $-\mathrm{Ca}^{+2}$ & $+\mathrm{Ca}^{+2}$ & & $-\mathrm{Ca}^{+2}$ & $+\mathrm{Ca}^{+2}$ & & $-\mathrm{Ca}^{+2}$ & $+\mathrm{Ca}^{+2}$ & \\
\hline & \multicolumn{9}{|c|}{ Starch\% (in fresh seeds) } \\
\hline 0 & 4.69 & 5.20 & 4.95 & 4.61 & 5.15 & 4.88 & 4.65 & 5.18 & 4.92 \\
\hline 100 & 4.88 & 5.52 & 5.20 & 4.73 & 5.40 & 5.07 & 4.81 & 5.46 & 5.14 \\
\hline 200 & 4.99 & 5.69 & 5.34 & 5.00 & 5.61 & 5.31 & 5.00 & 5.65 & 5.33 \\
\hline 300 & 5.31 & 5.83 & 5.57 & 5.24 & 5.86 & 5.55 & 5.28 & 5.85 & 5.56 \\
\hline 400 & 5.30 & 5.85 & 5.58 & 5.24 & 5.82 & 5.53 & 5.27 & 5.84 & 5.56 \\
\hline Mean (A) & 5.03 & 5.62 & & 4.96 & 5.57 & & 5.00 & 5.60 & \\
\hline $\mathrm{LSD}_{0.05} \mathrm{~A}$ & \multicolumn{3}{|c|}{0.15} & \multicolumn{3}{|c|}{0.13} & \multicolumn{3}{|c|}{0.14} \\
\hline B & \multirow{2}{*}{\multicolumn{3}{|c|}{$\begin{array}{l}0.23 \\
0.27\end{array}$}} & \multicolumn{3}{|c|}{0.19} & \multicolumn{3}{|c|}{0.21} \\
\hline AxB & & & & & 0.23 & & & 0.25 & \\
\hline \multicolumn{10}{|c|}{ Total soluble sugars\% (in fresh seeds) } \\
\hline 0 & 4.04 & 3.62 & 3.83 & 4.00 & 3.70 & 3.85 & 4.02 & 3.66 & 3.84 \\
\hline 100 & 3.90 & 3.45 & 3.68 & 3.85 & 3.55 & 3.70 & 3.88 & 3.50 & 3.69 \\
\hline 200 & 3.70 & 3.26 & 3.48 & 3.64 & 3.38 & 3.51 & 3.67 & 3.32 & 3.50 \\
\hline 300 & 3.38 & 3.08 & 3.23 & 3.43 & 3.12 & 3.28 & 3.41 & 3.10 & 3.26 \\
\hline 400 & 3.36 & 3.04 & 3.20 & 3.41 & 3.19 & 3.30 & 3.39 & 3.11 & 3.25 \\
\hline Mean (A) & 3.68 & 3.29 & & 3.67 & 3.39 & & 3.67 & 3.34 & \\
\hline LSD $_{0.05}$ A & & 0.09 & & & 0.11 & & & 0.10 & \\
\hline B & & 0.14 & & & 0.16 & & & 0.15 & \\
\hline AxB & & 0.18 & & & 0.19 & & & 0.19 & \\
\hline \multicolumn{10}{|c|}{ Protein \% (in fresh seeds) } \\
\hline 0 & 17.80 & 20.12 & \begin{tabular}{|l|}
18.96 \\
\end{tabular} & 16.98 & 19.86 & 18.42 & 17.39 & 19.99 & 18.69 \\
\hline 100 & 17.94 & 21.24 & 19.59 & 17.81 & 20.93 & 19.37 & 17.88 & 21.09 & 19.49 \\
\hline 200 & 18.81 & 22.10 & 20.46 & 18.63 & 22.06 & 20.35 & 18.72 & 22.08 & 20.40 \\
\hline 300 & 20.16 & 24.06 & \begin{tabular}{|l|}
22.11 \\
\end{tabular} & 20.20 & 24.21 & 22.21 & 20.18 & \begin{tabular}{|l|}
24.14 \\
\end{tabular} & 22.16 \\
\hline 400 & 20.13 & 24.10 & \begin{tabular}{|l|}
22.12 \\
\end{tabular} & 20.24 & 24.15 & 22.20 & 20.19 & 24.13 & 22.16 \\
\hline Mean (A) & 18.97 & 22.32 & & 18.77 & 22.24 & & 18.87 & \begin{tabular}{|l|}
22.29 \\
\end{tabular} & \\
\hline LSD $_{0.05} \quad \mathrm{~A}$ & & 0.42 & & & 0.61 & & & 0.52 & \\
\hline B & & 0.63 & & & 0.93 & & & 0.78 & \\
\hline AxB & & 0.79 & & & 1.11 & & & 0.95 & \\
\hline
\end{tabular}

\section{Conclusion}

Within the experimental conditions studied, it has been concluded that the work within hand gave an evidence to the role of $\mathrm{Ca}^{++}$(as a calcium paste covers the seeds before sowing) and ground sunflower heads (as a 
material, having high water holding capacity, by which seed-beds were fecundated before planting) besides, the role of ascorbic acid (as an antioxidant by which plant foliage sprayed especially at the concentration of $300 \mathrm{mgL}^{-1}$ ) in inducing salinity tolerance of pea plants cultivated in saltaffected reclaimed soils containing salts concentration at about $5000 \mathrm{ppm}$ leading to favourable growth and consequently obtain economic yield under such contitions.

\section{REFERENCES}

A.O.A.C. (1995). Association of Official Analytical Chemists. Official Methods of Analysis. $15^{\text {th }}$ ed., Washington, D. C., USA.

Abbas, M.A.; M.E. Younis and W.M. Shukry (1991). Plant growth, metabolism and adaptation in relation to stress conditions. III. Effect of salinity on the internal solute concentrations in Phaseolus vulgaris. J. Plant Physiol., 138: 722-729.

Abdel-Naby, A.; M.S. El-Beltagy; A.F. Abou-Hadid; Y.I. Helmy and S.O. ElAbd (2001). Effect of salinity stress modified by calcium application on potato plants. Egypt. J. Hort., 28(4): 519-530.

Ahmed, F.F.; O.H. Darwish; A.A. Gobara and A.H. Ali (1998). Growth, nutritional status and productivity of Flame seedless grapevine as influenced by the application of citrine and ascobine. Amer. Soc. Agron. Crops Sci., Soc. America, Soil Sci., p.252.

Ahmed, F.F.; O.H. Darwish; A.A. Gobara and A.H. Ali (2002). Physiological studies on the effect of ascorbic and citric acids in combined with some micronutrients on Flame seedless grapevine. Minia J. Agric. Res. \& Develop., 22(1): 105- 114.

Ahmed, F.F.; A.S. Abdalla and A.M.T. Sabour (2003). Growth and fruiting of williams banana as affected by some antioxidant and biofertilizer treatments. Minia J. Agric. Res.\&Develop., 23(1): 51-68.

Ahmed, F.F. and H.M. Abd El-Hameed (2004). Influence of some antioxidants on growth, vine nutritional status, yield and quality of berries in banaty grapevines. Assiut J. Agric. Sci., 35(4): 131- 140.

Al-Qubaie, A.I. (2002). Response of Ficus nitida L. seedlings to the application of some antioxidants under soil salinity conditions. Minia J. Agric. Res.\&Develop., 22 (3): 235- 254.

Ali, A.H. (2000). Response of Flame seedless grapevines to spraying with ascorbic acid and boron. Minia J. Agric. Res.\&Develop., 20 (1): 159174.

Ali, Z.A. (2002). Effect of foliar application with ascorbic acid on vegetative growth and some biochemical constituents of tomato plants. J. Agric. Sci., Mansoura Univ., 27(10): 6765- 6775.

Arnon, D.I. (1949). Copper enzymes in isolated chloroplasts. Polyphenoloxidase in Beta vulgaris L. Plant Physiol., 24: 1-5.

Arrigoni, O. (1994). Ascorbate system in plant development. J. Bioenerg. Biomembr., 26: 407- 419. 
Bates, L.S.; R.P. Waldren and I.D. Teare (1973). Rapid determination of free proline for water stress studies. Plant and Soil, 39: 205-207.

Chapman, H.D. and P.F. Pratt (1961). Methods of Analysis for Soil. Plants and Water, Univ. Calif., D.V., Agric. Sci., USA.

Cordoba-Pedregosa, M.C.; J.A. Gonzalez-Reyes; M.S. Sandillas; P. Navas and F. Cordoba (1996). Role of apoplastic and cell-wall peroxidases on the stimulation of root elongation by ascorbate. Plant Phyiol., 112: 1119- 1125.

Cramer, G.R.; A. Lauchli and V.S. Polito (1985). Displacement of $\mathrm{Ca}^{2+}$ by $\mathrm{Na}^{+}$ from the plasmalemma of root cell. Plant Phyiol., 79: 207.

Dubois, M.F.; K.A. Gilles; J.K. Hamiton; P.A. Robers and F. Smith (1956). Colorimetric methods for determination of sugars and related substances. Anal. Chem., 28: 350-354.

Eastwood, M.A.(1973). Methods for determining physical characteristics of fiber. Cereal Foods World Abstracts, 21: 426.

El-Saidi, M.T. (1997). Salinity and its effect on growth, yield and some physiological processes of crop plants. In: Strategies for improving salt tolerance in higher plants. Jaiwal, P.K.; R.P. Singh and Anju Gulati (Eds.). Oxford and IBH Publishing Co. Pvt. Ltd. New Delhi, Calcutta Enfield (USA), pp.111- 127.

Elade, Y. (1992). The use of antioxidants to control gray mould (Botrytis cineria) and white mould (Sclerotinia sclerotiorum) in various crops. Plant Pathol., 141: 417- 426.

Epstein, E. (1961). The essential role of calcium in selective cations transports by plant cells. Plant Phyiol., 36: 437.

Farag, K.M. (1996). Use of urea, phenylalanine, thiamine or their combinations to accelerate anthocyanins development and their effect on the storage life of Flame seedless grapes. First Egyptian Hungarian Hort. Conf., Kafr El-Sheikh, Egypt, 15-17 Sept.

Farago, S. and C. Brunhold (1994). Regulation of thiol contents in maize roots by intermediates and effectors of glutathione synthesis. J. Plant Physiol., 144: 433- 437.

Foyer, C.H. and B. Halliwell (1976). The presence of glutathione and glutathione reductase in chloroplasts: A proposed role in ascorbic acid metabolism. Planta, 157: 239- 244.

Foyer, C.H.; R.T. Furbank; J. Harbinson and P. Horton (1990). The mechanism contributing to photosynthetic control of electron transport by carbon assimilation in leaves. Photosynth. Res., 25: 83- 100.

Gonzalez-Reyes, J.A.; F.J. Alcain; J.A. Caler; A. Serrano; F. Cordoba and P. Navas (1994). Relationship between apoplastic ascorbate regeneration and stimulation of root growth in Allium cepa L. Plant Sci., 100: 23-29.

Greenway, H.R. and R. Munns (1980). Mechanisms of salt tolerance in nonhalophytes. Annu. Rev. Plant Physiol., 31: 149.

Gupta, P.K.; A.L. Nadgir; A.F. Macarentias and V. Jagannathan (1980). Tissue culture of forest trees: Clonal multiplication of Tecoma grandis L. (treak) by tissue culture. Plant Sci. Letters, 17: 259-268. 
Hafez, A.R. and D.S. Mikkelsen (1981). Colorimetric determination of nitrogen for evaluating the nutritional status of rice. Commun. Soil Sci. and Plant Analysis, 12(1): 61-69.

Hanson, J.B. (1984). The function of calcium in plant nutrition. In: Tinker, P.B. and A. Lauchi (Eds.) Advances in "Plant Nutrition", vol. 1, Praeger, New York, pp. 149-208.

Jackson, M.L. (1967). Soil Chemical Analysis. Prentice-Hall of India Private Limited, New Delhi, pp.144-197 and 326- 338.

Kahlan, T.S.; F. I. Chaw; J. L. Hoefer and A.A. Betschart (1986). Bioavailability of vitamins $A$ and $E$ as influenced by wheat bran and bran particle size. Cereal Chem.., 63: 490.

Klopfenstein, C.F. (1990). Nutritional properties of coarse and fine sugar beet fiber and hard red wheat bran. 2. Effect on calcium and iron utilization. Cereal Chem., 67: 542.

LaHaye, P.A. and E. Epstein (1969). Salt tolerance by plants: enhancement with calcium. Science, 166: 395.

Leopold, A.C. and R.P. Willing (1984). Evidence for toxicity effects of salt on membranes. In: Staples, R.C. and Toenniessen (Eds.). Salinity tolerance in plants: Strategies for crop improvement. John Wiley and Sons, New York, 67.

Maas, E.V. (1986). Crop tolerance to saline soil and water. Proc. US. Pak. Biosaline Res. Workshop, Botany Dept., Karachi Univ., Pakistan, pp. 205- 219.

Mongeau, R. and R. Brassard (1982). Insoluble dietary fiber from breakfast cereals and brans: bile salt binding and water holding capacity in relation to particle size. Cereal Chem., 59: 413.

Mostafa, E.A.M. (2004). Effect of spraying with ascorbic acid, vitamin B and active dry yeast on growth, flowering, leaf mineral status, yield and fruit quality of Grand nain banana plants. Annals Agric. Sci., Ain Shams Univ., Cairo, 49(2): 643- 659.

Navas, P. and C. Gomez-Diaz (1995). Ascorbate free radical and its role in growth control. Protoplasm, 184: 8-13.

Page, A.I.; R.H. Miller and D.R. Keeny (1982). Methods of Soil Analysis. Part II. Chemical and Microbiological Methods. $2^{\text {nd }}$ ed. Amer. Soc. Agron., Madison, Wisconsin, USA.

Pasternak, D. (1987). Salt tolerance and crop production. A comprehensive approach. Ann. Rev. Phytopathol., 20: 271-291.

Piper, C.S. (1947). "Soil and Plant Analysis". The University of Adelaide, Adelaide, UK.

Poovaiah, B.W. and A.S.N. Reddy (1993). Calcium and signal transduction in plants. CRC Crit. Rev. Plant Sci., 12(3): 185.

Prosky, L.; N.G. Asp; T.F. Scheizer; J.W. Devries and I. Furda (1988). Determination of insoluble, soluble and total dietary fiber in foods and food products. Interlaboratory study. J. Am. Anal. Chem., 71: 1017.

Prusky, D. (1988). The use of antioxidants to delay the onset of anthracnose and stem end decay in avocado fruits after harvest. Plant Disease, 72: 381- 384. 
Rady, M.M. (2006). Efficiency of growth and productivity of sunflower plants as affected by ascorbic acid under saline reclaimed soil conditions. $2^{\text {nd }}$ Conference on Farm Integrated Pest Management, Fac. Agric., Fayoum Univ., Fayoum, Egypt, pp. 186- 200.

Ragab, M.M. (2002). Effect of spraying urea, ascorbic acid and NAA on fruiting of Washington Navel orange trees. M.Sc. Thesis, Fac. Agric., Minia Univ., Egypt.

Rautenkranz, A.; F. Machler; E. Martinoia and J. Oerti (1994). Transport of ascorbic and dehydroascorbic acids across protoplast and vacuole membranes isolated from barley (Hordeum vulgare L. cv. Gebrel) leaves. Plant Physiol., 106: 187- 193.

Robinson, F.A. (1973). Vitamins "Phytochemistry", Vol. III: 195-198, Lawrence P. Miller (Ed.), Van Nostrand Reinhold Co., New York.

Scandalios, J.G. (1997). Molecular genetics of superoxide dismutases in plants. pp.527- 568. In: Scandalios, J.G. (ed.). Oxidative stress and the molecular biology of antioxidant defenses. Cold Spring Harbor Lab. Press, Plainview, N.Y.

Schneeman, B.O. (1986). Dietary fiber. Physical and chemical properties, methods of analysis, and physiological effects. Food Technol., 40: 104.

Schneeman, B.O. (1989). Dietary fiber. A Scientific Status Summary by The Institute of Food Technologists Expert Panel on Food Safety and Nutrition. Food Technol., 41: 133.

Shahidi, F. and P.K. Wanasundara (1992). Phenolic-antioxidants. Crit. Rev. Food Sci. Nutr., 32: 67- 103.

Snedecor, W.C. and W.G. Cochran (1980). Statistical Methods. $7^{\text {th }}$ ed., lowa State Univ. Press, Ames, lowa, USA.

Snell, F.D. and C.T. Snell (1953). Colorimetric methods of analysis including some turbimetric and morpholometric methods. D. Van-Nastrod Co., Inc. New Jersey, Toronto, New York, London, vol. III, p. 606.

Tarraf, S.A.; K.M. Gamal El-Din and L.K. Balbaa (1999). The response of vegetative growth, essential oil of lemongrass (Cymbopogon citratus Hort.) to foliar application of ascorbic acid, nicotinamide and some micronutrients. Arab. Univ. J. Agric. Sci., Ain Shams Univ., Cairo, 7(1): 247- 261.

Wise, R.R. and A.W. Naylor (1987). The peroxidative destruction of lipids during chilling injury to photosynthesis and ultrastructure. Plant Physiol., 83: 272- 277.

Zhang, X. and R.E. Schmidt (2000). Hormone-containing products impact on antioxidant status of tall fescue and creeping bentgrass subjected to drought. Crop Sci., 40: 1344- 1349. 
تأثير بعض المعاملات على النمو والقدرة المحصولية لنباتـات البسلة النامية تحت

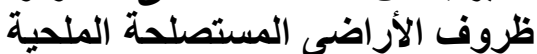

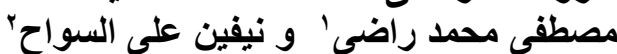

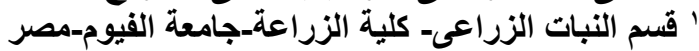

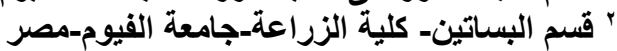

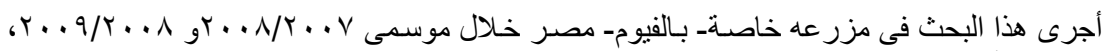
وذللك بهذف دراسة تأثير تغليف البذور بعجينة الكالبيوم وزر اعتها فئ مر اقد تحتوى على على كمية كافية من مخلفات

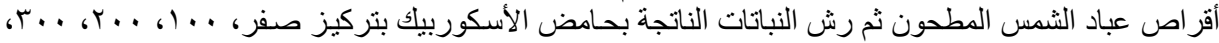

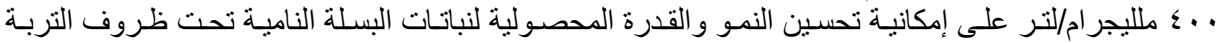

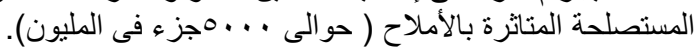

وتثير النتائج المتحصل عليها إلى: إئى







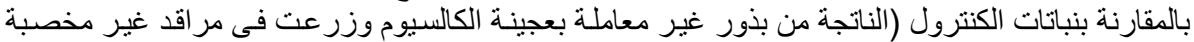

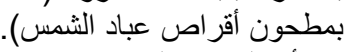

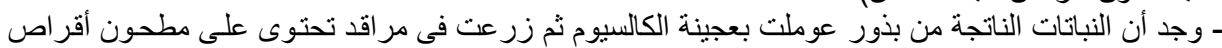

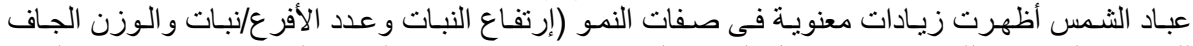

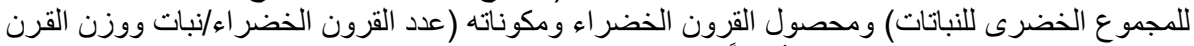

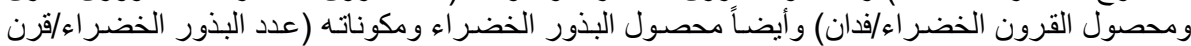

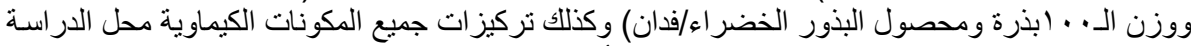

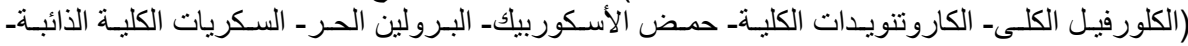



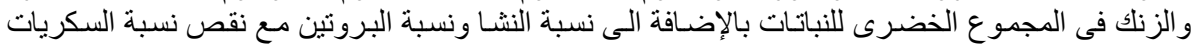
الكلية الذائبة في البذور الخيوعة الخضر اءع).



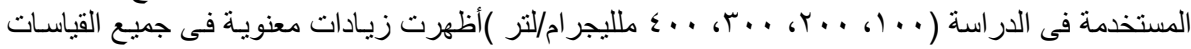

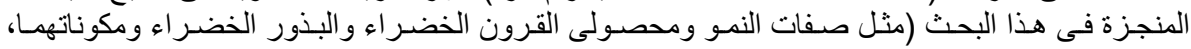

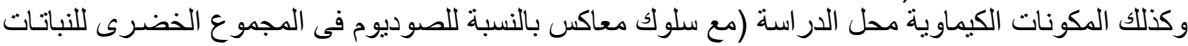

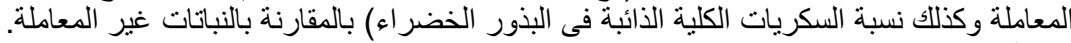



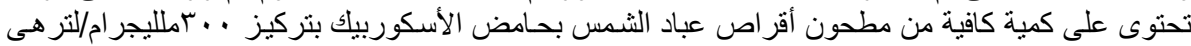

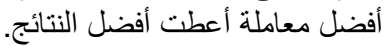

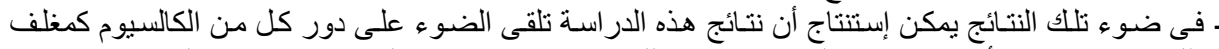



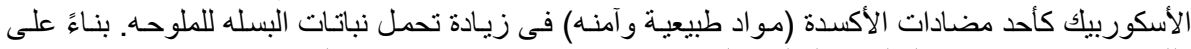

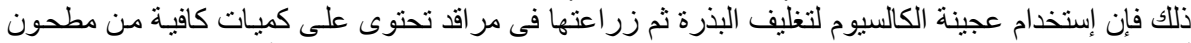

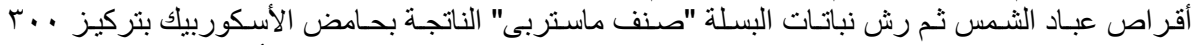

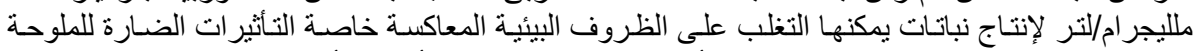





